\title{
calcul au cisaillement du sable sollicité en déformation plane
}

\author{
par \\ J. Monnet \\ Assistant \\ Laboratoire de Géotechnique de I'INSA de Lyon
}

\begin{abstract}
La détermination classique du comportement effortdéformation d'un sol est réalisée au laboratoire par des ssais triaxiaux à symétrie axiale de révolution. Cependant, dans un grand nombre de cas, on adopte en mécanique des sols, l'hypothèse de la déformation plane et on ramène l'étude du comportement du massif à celle d'un milieu à deux dimensions (soutènements, fondations et talus linéaires...) tout en continuant à caractériser le sol par des paramètres mécaniques déterminés par les essais triaxiaux classiques.
\end{abstract}

Le développement de moyens sophistiqués de calcul par la méthode des éléments finis impose de tendre à une cohérence entre les hypothèses de calcul adoptées et les lois expérimentales traduisant le comportement mécanique des sols.

Les modèles théoriques exprimant le comportement du sol en déformation plane et utilisables par un ordinateur sont dûs à Duncan et Chang (1970), Simpson et Worth (1972), Lade et Duncan (1975), Boulon, Chambon et Darve (1977), Vermeer (1978), Simpson, O'Riordan et Croft (1979). Ils nécessitent l'introduction de 7 à 13 paramètres selon le cas. Pour le praticien, plus ce nombre est réduit, plus l'utilisation est facilitée.

Nous présentons ici une loi à 4 paramètres pour le sol pulvérulent. Nous sommes partis des travaux de Frydman, Zeitlen, Alpan (1973) qui ont recherché la relation liant, au cours du cisaillement, les trois contraintes principales aux trois déformations plastiques principales, en exprimant la conservation de l'énergie pendant le phénomène. Monnet (1977) et Monnet, Gielly (1979) appliquent cette formulation au calcul par la méthode des éléments finis. Ils constatent que les résultats du calcul par ordinateur correspondent bien aux données expérimentales pour l'essai triaxial. Nous présentons une extension du domaine d'utilisation de notre modèle au cas du chargement en déformation plane et comparons sa formulation à celle de Rowe. Nous l'utilisons dans un calcul par la méthode des éléments finis, d'essais de cisaillement en déformation plane et comparons les résultats théoriques et expérimentaux.
Nous adopterons comme convention de signe, celle de la résistance des matériaux (les contraintes et les éformations sont négatives en compression) pour pouvoir appliquer directement les formules trouvées au programme de calcul par éléments finis.

\section{Théorie du comportement du sol en défor- mation plane au cisaillement}

Il est supposé que le sol se comporte de trois façons différentes au fur et à mesure que le cisaillement augmente. II commence par réagir élastiquement, puis à partir d'un point appelé seuil d'écrouissage, il se produit un écrouissage durcissant. Enfin, le cisaillement maximum est atteint pour la valeur donnée par le critère de Mohr-Coulomb et il se produit alors un écoulement plastique parfait "non standard".

\subsection{Comportement du sol en élasticité}

II est donné par les relations classiques de la loi de Hooke et nécessite la connaissance du module de Young et du coefficient de Poisson.

\subsection{Comportement du sol en écrouissage durcissant}

Ce comportement commence quand le seuil d'écrouissage est atteint, ce qui correspond à la relation :

$$
-\frac{\tau_{\text {oct }}}{\sigma_{\text {oct }}}=\operatorname{tg} \phi_{\mu}
$$

avec

$$
\begin{aligned}
& \tau_{\text {oct }}=\frac{1}{3} \sqrt{\left(\sigma_{1}-\sigma_{2}\right)^{2}+\left(\sigma_{2}-\sigma_{3}\right)^{2}+\left(\sigma_{3}-\sigma_{1}\right)^{2}} \\
& \sigma_{\text {oct }}=\frac{1}{3}\left(\sigma_{1}+\sigma_{2}+\sigma_{3}\right)
\end{aligned}
$$

$\sigma_{1}, \sigma_{2}, \sigma_{3}$ sont les contraintes principales négatives en compression.

$\sigma_{\text {oct }}$ est appelée aussi contrainte moyenne

$\phi_{\mu}$ est l'angle de frottement intergranulaire 
A partir de ce seuil, les déformations sont considérées comme totalement plastiques. Pendant cette phase du cisaillement, on observe une augmentation de volume du sol.

Frydman et al. (1973) ont montré que la conservation de l'énergie de déformation plastique conduit la relation :

$$
-\frac{\tau_{\text {oct }}}{\sigma_{\text {oct }}}=\operatorname{tg} \phi_{\mu}+\frac{d \varepsilon_{\text {oct }}^{\text {pot }}}{d \varepsilon_{\text {oct }}^{\prime p}}
$$

avec

$$
\begin{aligned}
d \varepsilon_{\text {oct }}^{p} & =\frac{1}{3}\left(d \varepsilon_{1}^{p}+d \varepsilon_{2}^{p}+d \varepsilon_{3}^{p}\right) \\
d \varepsilon_{\text {oot }}^{\prime} & =\frac{1}{3} \sqrt{\left(d \varepsilon_{1}^{p}-d \varepsilon_{2}^{p}\right)^{2}+\left(d \varepsilon_{2}^{p}-d \varepsilon_{3}^{p}\right)^{2}+\left(d \varepsilon_{3}^{p}-d \varepsilon_{1}^{p}\right)^{2}}
\end{aligned}
$$

$\varepsilon_{1}^{p}, \varepsilon_{2}^{p}, \varepsilon_{3}^{p}$ déformations plastiques principales.

La relation (2) ne permet pas de calculer directement le comportement du sol, et il est nécessaire de pousser plus loin l'analyse pour aboutir à des calculs théoriques par éléments finis. II faut d'abord définir la direction de la composante d $\varepsilon^{\prime}{ }^{\prime}$ ct du vecteur déformation plastique dans le plan octaédrique, ce qui permettra ensuite, grâce à la relation (2), de trouver la direction de la déformation dans l'espace des contraintes. Enfin il faudra trouver la longueur de la déformation plastique. Nous faisons l'hypothèse de la coîncidence des axes de contraintes principales et d'incréments de déformations plastiques principales.

\subsubsection{Direction de la composante $d \varepsilon^{\prime p}$ oct du vecteur déformation plastique}

Monnet (1977) et Monnet et al. (1979) ont montré que la relation (2) dans le cas de la symétrie axiale conduit à :

$$
\left\{d \varepsilon^{p}\right\}=d \lambda\left\{\begin{array}{l}
a \\
b \\
c
\end{array}\right\}
$$

$$
\begin{aligned}
& a=-\frac{\frac{\sqrt{2}}{\sqrt{3}} \cdot \cos \gamma}{\frac{\tau_{\text {oct }}}{\sigma_{\text {oct }}}+\operatorname{tg} \phi_{\mu}}+\frac{1}{\sqrt{3}} \\
& b=-\frac{-\frac{1}{\sqrt{6}} \cos \gamma+\frac{1}{\sqrt{2}} \sin \gamma}{\frac{\tau_{\text {oct }}}{\sigma_{\text {oct }}}+\operatorname{tg} \phi_{\mu}}+\frac{1}{\sqrt{3}} \\
& c=-\frac{-\frac{1}{\sqrt{6}} \cos \gamma-\frac{1}{\sqrt{2}} \sin \gamma}{\frac{\tau_{\text {oct }}}{\sigma_{\text {oct }}}+\operatorname{tg} \phi_{\mu}}+\frac{1}{\sqrt{3}}
\end{aligned}
$$

où $\gamma$ est l'angle qui définit la direction du cisaillement ctaédrique tel que :

$$
\operatorname{tg} \gamma=\frac{\sqrt{3}\left(\sigma_{2}-\sigma_{3}\right)}{2 \sigma_{1}-\sigma_{2}-\sigma_{3}}
$$

Dans le cas de la déformation plane, la démonstration de (3) reste valable.

Cependant nous supposerons que la distorsion plastique $d \varepsilon_{\text {oct }}^{\prime p}$ n'est plus colinéaire au cisaillement octaédrique. Nous appellerons $\gamma^{\prime}$ l'angle qui permet de trouver sa direction:

$$
\operatorname{tg} \gamma^{\prime}=\frac{\sqrt{3}\left(\mathrm{~d} \varepsilon_{2}^{p}-\mathrm{d} \varepsilon_{3}^{p}\right)}{2 \mathrm{~d} \varepsilon_{1}^{p}-\mathrm{d} \varepsilon_{2}^{p}-\mathrm{d} \varepsilon_{3}^{p}}
$$

En déformation plane, il existe une direction de déformation nulle, qui correspond à l'axe de contrainte principale moyenne. On a donc :

$$
\mathrm{b}=-\frac{-\frac{1}{\sqrt{6}} \cos \gamma^{\prime}+\frac{1}{\sqrt{2}} \sin \gamma^{\prime}}{\frac{\tau_{\text {oct }}}{\sigma_{\text {oct }}}+\operatorname{tg} \phi_{\mu}}+\frac{1}{\sqrt{3}}=0
$$

Si on pose $t=\operatorname{tg} \frac{\gamma^{\prime}}{2}$

et

$$
\operatorname{tg} \delta=\frac{1}{\frac{\tau_{\text {oct }}}{\sigma_{\text {oct }}}+\operatorname{tg} \phi_{\mu}}
$$

il vient alors :

$$
t^{2}\left(\frac{1}{\operatorname{tg} \delta \cdot \sqrt{3}}+\frac{1}{\sqrt{6}}\right)+t \cdot \sqrt{2}+\frac{1}{\operatorname{tg} \delta \cdot \sqrt{3}}-\frac{1}{\sqrt{6}}=0
$$

qui se résoud par le discriminant :

$$
\Delta=4\left[\frac{2}{3}-\frac{1}{\operatorname{tg}^{2} \delta \cdot 3}\right]
$$

et dont les solutions sont :

$$
\operatorname{tg} \frac{\gamma^{\prime}}{2}=\frac{-\sqrt{2} \pm \sqrt{\Delta}}{2\left(\frac{1}{\operatorname{tg} \delta \cdot \sqrt{3}}+\frac{1}{\sqrt{6}}\right)}
$$

Pour choisir entre les deux valeurs de $\gamma^{\prime}$, il faut emarquer que la distorsion plastique $\mathrm{d} \varepsilon^{\prime}{ }_{\mathrm{oct}}$ sera dans la même moitié de plan que le cisaillement octaédrique $\tau_{\text {oct }}$ donc que $\gamma^{\prime}$ sera du signe de $\sigma_{2}-\sigma_{3}$.

\subsubsection{Direction de la déformation plastique dans l'espace des contraintes}

L'angle $\gamma^{\prime}$ étant alors défini par (6), on aura la direction de la déformation plastique par:

$$
\left\{d \varepsilon^{p}\right\}=d \lambda\left\{\begin{array}{l}
a \\
o \\
c
\end{array}\right\}=d \lambda\{v\}
$$

avec :

et

$$
\begin{gathered}
\mathrm{a}=-\frac{\frac{\sqrt{2}}{\sqrt{3}} \cdot \cos \gamma^{\prime}}{\frac{\tau_{\text {oct }}}{\sigma_{\text {oct }}}+\operatorname{tg} \phi_{\mu}}+\frac{1}{\sqrt{3}} \\
\mathrm{c}=-\frac{-\frac{1}{\sqrt{6}} \cos \gamma^{\prime}-\frac{1}{\sqrt{2}} \sin \gamma^{\prime}}{\frac{\tau_{\text {oct }}}{\sigma_{\text {oct }}}+\operatorname{tg} \phi_{\mu}}+\frac{1}{\sqrt{3}}
\end{gathered}
$$

1.2.3 Longueur de la déformation plastique d'écrouissage

Le vecteur $\{v\}$ dans la relation (7) étant connu en fonction des contraintes, on trouve la valeur du scalaire $\mathrm{d} \lambda$, donc de la déformation plastique par la relation classique :

$$
\begin{aligned}
\mathrm{d} \lambda & =\frac{f(\sigma, k)}{\{v\}^{\top}[E]\left\{\frac{\partial f}{\partial \sigma}\right\}-\{v\}^{\top}\left\{\frac{\partial k}{\partial\left(d \varepsilon^{p}\right)}\right\} \frac{\partial f}{\partial k}} \\
k & =\frac{\varepsilon_{\text {oct }}^{p}}{\varepsilon_{\text {oct }}^{\prime p}}
\end{aligned}
$$

et $\quad f(\sigma, k)=\tau_{\text {oct }}+\sigma_{\text {oct }}\left(\operatorname{tg} \phi_{\mu}+k\right)$ 
qui exprime la fonction de charge du matériau. Le terme $k$ dépend des déformations plastiques d'écrouissage, c'est-à-dire des déformations totales se produisant à partir du seuil défini par la formule (1). Cela suppose que la formule (2) est également valable en déformations plastiques principales totales.

Pour résoudre le problème dans un calcul par éléments finis, il suffit alors d'appliquer la méthode des contraintes initiales en utilisant les relations définies auparavant.

\subsection{Comportement du sol en écoulement plasti- que parfait "non standard"}

La fonction de charge qui définit l'état limite du sol est le critère de Mohr-Coulomb :

$$
g(\sigma)=\sigma_{3}-\sigma_{1}+\sin \phi\left(\sigma_{1}+\sigma_{3}\right)
$$

où $\sigma_{1}$ est la contrainte de compression maximum, et

$\sigma_{3}$ est la contrainte minimum.

Nous faisons l'hypothèse que les déformations plastiques changent d'état quand on arrive à l'état ultime. A partir du moment où la fonction $\mathrm{g}$ s'annule, nous considérerons qu'il se produit des déformations plastiques ultimes différentes des déformations plastiques d'écrouissage. Ce sont ces nouvelles déformations plastiques qui seront utilisées dans la suite. La conservation de l'énergie de déformation implique alors que la relation (2) soit vraie pour ces nouvelles déformations.

\subsubsection{Direction de la déformation plastique dans l'espace des contraintes}

L'analyse effectuée précédemment pour l'écrouissage est également valable dans le cas de l'équilibre limite. La déformation plastique est donc définie en direction par la relation (7).

\subsubsection{Longueur de la déformation plastique ultime}

On montre facilement que la longueur de cette déformation est proportionnelle à $d \lambda$ tel que :

$$
\mathrm{d} \lambda=\frac{g(\sigma)}{\{v\}^{\top}[E]\left\{\frac{\partial g}{\partial \sigma}\right\}} .
$$

La méthode des contraintes initiales permet alors de résoudre le calcul du sol à l'état ultime de cisaillement.

\section{Comparaison entre la théorie de Rowe ainsi que notre théorie avec plusieurs essais de laboratoire}

\subsection{Les essais utilisés}

Nous avons choisi de comparer les résultats donnés par notre analyse théorique ainsi que l'analyse de Rowe (1962) avec des résultats expérimentaux puisés dans la littérature. Les premiers essais de laboratoire en déformation plane, pour lesquels on mesure les trois contraintes principales $\left(\sigma_{1}, \sigma_{2}, \sigma_{3}\right)$ et les deux déformations $\left(\varepsilon_{1}, \varepsilon_{3}\right)$ sont ceux de Cornforth (1964). Par la suite, on trouve les travaux de Lee (1970), Lade (1972), Al-Hussaini (1973), Ichihara et al. (1973) qui utilisent du sable homogène. Tous constatent que le sable se dilate moins et possède un angle de frottement ultime de 1 à 4 degrés plus fort qu'en symétrie axiale. Nous utiliserons les travaux de Cornforth (1964) et Al-Hussaini (1973). Ces deux auteurs travaillent sur le même type d'appareillage. L'échantillon est un prisme de $40,6 \mathrm{~cm}$ de long (16 inches), 5,1 cm de large (2 inches) et $10,2 \mathrm{~cm}$ de haut ( 4 inches).

Les deux faces distantes de $40,6 \mathrm{~cm}$ sont maintenues parallèles pendant l'essai pour donner la direction de déformation nulle longitudinalement. Les deux faces distantes de 10,2 cm sont maintenues par des plaques rigides et lisses et permettent de charger l'échantillon verticalement. Les deux faces distantes de $5,1 \mathrm{~cm}$ sont recouvertes d'une gaine étanche et libre de se déformer horizontalement. Elles subissent la pression de l'eau de l'enceinte qui entoure tout l'appareillage. C'est la seule direction pour laquelle les déformations peuvent ne pas être homogènes.

La mise en équilibre limite du sol avec l'hypothèse de Mohr-Coulomb indique que la ligne de glissement générale fait un angle de $\frac{\pi}{4}-\frac{\phi}{2}$ avec la direction de contrainte principale majeure. On doit donc veiller, quand on fait un essai, à ce que le sol soit libre de se déplacer le long de cette ligne. Pratiquement, cela implique la relation:

$$
\operatorname{tg}\left(\frac{\pi}{4}-\frac{\phi}{2}\right)>\frac{\text { largeur }}{\text { hauteur }} \text { de l'échantillon et } \phi<36,9^{\circ} \text {. }
$$

Cette condition n'est pas à vérifier si la lubrification des plaques est bonne. Nous utiliserons donc dans un premier temps ces résultats expérimentaux sans en tenir compte.

Nous exploiterons également les résultats de Lade (1972) pour lequel l'échantillon est un cube de $7,6 \mathrm{~cm}$ de côté. Chaque face est chargée par une plaque rigide et-lubrifiée, ce qui fait que les déformations sont homogènes dans les trois directions principales. L'échantillon est montré sur une presse qui peut faire varier les trois contraintes $\sigma_{1}, \sigma_{2}, \sigma_{3}$. Les essais de déformation plane ont été réalisés en maintenant la contrainte intermédiaire $\sigma_{2}$ telle qu'elle annule les déformations de l'échantillon dans cette direction.

\section{2 Étude de l'écrouissage durcissant du sable}

Nous considérons la déformation plastique d'écrouissage expérimentale comme la différence de déformation entre le moment où le sol est à son volume minimum et celui oủ il atteint le cisaillement maximum (colonnes 4 et 5 des tableaux 1 et 2 ).

\subsection{1 Étude de la direction de la distorsion} plastique $d \varepsilon_{o c t}^{\prime \circ}$ dans le plan octaédrique

\subsubsection{Direction expérimentale}

Connaissant les déformations plastiques expérimentales on peut, grâce à la formule (5), trouver la valeur de l'angle $\gamma^{\prime}$ (colonne 7 du tableau 1). On connaît également les contraintes au moment du maximum de cisaillement, ce qui permet de calculer la direction du cisaillement octaédrique dans le plan, grâce à la formule (4) qui donne $y$ (colonne 6 du tableau 1). Nous constatons alors un écart moyen de $16,8^{\circ}$, ce qui montre que l'hypothèse de la colinéarité de la distorsion plastique et du cisaillement octaédrique ne peut être retenue dans le cas de la déformation plane. 


\begin{tabular}{|c|c|c|c|c|c|c|c|c|c|c|c|c|c|}
\hline Essai & $\begin{array}{ll}\sigma_{1} & \\
\mathrm{kPa} & \\
& 1\end{array}$ & $\begin{array}{ll}\sigma_{2} & \\
\mathrm{kPa} & \\
& 2\end{array}$ & $\begin{array}{r}\sigma_{3} \\
\mathrm{kPa} \\
\\
\\
3\end{array}$ & 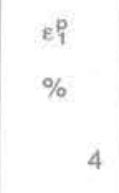 & $\begin{array}{ll}\varepsilon_{3}^{\mathrm{g}} & \\
\% & \\
& \\
& 5\end{array}$ & \begin{tabular}{c}
\multicolumn{1}{c}{$\gamma$} \\
degré \\
\\
6
\end{tabular} & $\begin{array}{c}\gamma^{\prime} \\
\text { degré } \\
\text { exp. } \\
1 \quad 7\end{array}$ & $\frac{\tau_{\text {oct }}}{\sigma_{\text {oct }}}$ & $\begin{array}{r}\begin{array}{c}\phi_{\mu} \\
\text { degré }\end{array} \\
9\end{array}$ & \begin{tabular}{c}
\multicolumn{1}{c}{$\phi_{c v}$} \\
degré \\
10
\end{tabular} & $\begin{array}{c}\gamma^{\prime} \\
\text { théorique } \\
\text { degré } \\
11\end{array}$ & 12 & $\begin{array}{c}\gamma^{\prime} \\
\text { Rowe } \\
\text { degré } \\
13\end{array}$ \\
\hline $\begin{array}{l}\text { LADE } \\
\text { CT1-L2 }\end{array}$ & $-313,8$ & $-162,3$ & -60 & -3.68 & 4,31 & $-156,4^{\circ}$ & $-147,4^{\circ}$ & $-0,583$ & 26,4 & $41,3^{\circ}$ & $-146,5^{\circ}$ & 5,230 & $-148,9^{\circ}$ \\
\hline $\begin{array}{l}\text { LADE } \\
\text { CTI-D2 }\end{array}$ & -703 & -280 & -60 & $-1,021$ & 1,594 & $-160,2^{\circ}$ & $-142,8^{\circ}$ & $-0,767$ & 26,4 & $41,3^{\circ}$ & $-139,0^{\circ}$ & 11,717 & $-136,6^{\circ}$ \\
\hline CORNFORTH & -1154 & -493 & -276 & $-1,00$ & 1,11 & $-166,3^{\circ}$ & $-148.2^{\circ}$ & $-0,583$ & 26,6 & $33,2^{\circ}$ & $-146,7^{\circ}$ & 4,181 & $-146,7^{\circ}$ \\
\hline $\begin{array}{l}\text { AL-HUSSAINI } \\
\text { A }\end{array}$ & -2207 & -876 & -483 & $-3,03$ & 3,53 & $-167,4^{\circ}$ & $-147.5^{\circ}$ & $-0,621$ & 27,4 & $33,3^{\circ}$ & $-147.6^{\circ}$ & 4,569 & $-145,3^{\circ}$ \\
\hline $\begin{array}{l}\text { AL-HUSSAINI } \\
\text { B }\end{array}$ & -1924 & -800 & -483 & $-0,43$ & 0.47 & $-167,9^{\circ}$ & $-148.5^{\circ}$ & -0.578 & 27,4 & $33,3^{\circ}$ & $-149,0^{\circ}$ & 3,983 & $-147,6^{\circ}$ \\
\hline
\end{tabular}

Tableau 1 Etude de la position du vecteur distorsion plastique et du cisaillement dans le plan octaédrique pendant l'écrouissage

\begin{tabular}{|c|c|c|c|c|c|c|c|c|c|c|c|c|c|}
\hline Essai & $\begin{array}{c}\sigma_{1} \\
\mathrm{kPa} \\
\\
\\
\\
1\end{array}$ & $\begin{aligned} \begin{array}{r}\sigma_{2} \\
\mathrm{kPa}\end{array} & \\
& 2\end{aligned}$ & $\begin{array}{l}\sigma_{3} \\
\mathrm{kPa} \\
\quad 3\end{array}$ & $\begin{array}{ll}\varepsilon_{1}^{p} & \\
\% & \\
& 4\end{array}$ & $\begin{array}{ll}\varepsilon ? & \\
\% & \\
& 5\end{array}$ & $\begin{array}{l}\frac{T_{\text {oct }}}{\sigma_{\text {oct }}} \\
6\end{array}$ & $\begin{array}{l}\frac{\varepsilon_{o c t}^{p}}{\varepsilon_{o c t}^{\prime}} \\
\% \\
\exp \\
\quad 7\end{array}$ & $\begin{array}{r}\phi_{\mu} \\
\text { degré } \\
8\end{array}$ & $\begin{array}{c}\frac{\varepsilon_{\mathrm{oct}}^{p^{\prime}}}{\varepsilon_{\mathrm{oct}}^{\prime p}} \\
\text { théorique } \\
9\end{array}$ & 10 & $\begin{array}{c}\phi_{\mathrm{cv}} \\
\text { degré } \\
11\end{array}$ & 12 & $\begin{array}{l}\frac{\varepsilon_{\text {oct }}^{p}}{\varepsilon_{\text {oct }}^{\prime}} \\
\text { Rowe } \\
13\end{array}$ \\
\hline $\begin{array}{l}\text { LADE } \\
\text { CT1-L2 }\end{array}$ & $-313,8$ & $-162,3$ & -60 & $-3,68$ & 4,31 & $-0,583$ & 0.064 & $26.4^{\circ}$ & 0.087 & 5,230 & $41,3^{\circ}$ & 1,071 & 0,028 \\
\hline $\begin{array}{l}\text { LADE } \\
\text { CT1-D2 }\end{array}$ & -703 & -280 & -60 & $-1,02$ & 1,594 & -0.767 & 0,176 & $26,4^{\circ}$ & 0,271 & 11.717 & $41,3^{\circ}$ & 2.400 & 0,327 \\
\hline CORNFORTH & -1154 & -493 & -276 & $-1,00$ & 1,11 & $-0,583$ & 0,043 & $26,6^{\circ}$ & 0,082 & 4,181 & $33,2^{\circ}$ & 1,222 & 0,082 \\
\hline $\begin{array}{l}\text { AL-HUSSAINI } \\
\text { A }\end{array}$ & -2207 & -876 & -483 & $-3,03$ & 3,53 & $-0,621$ & 0,062 & $27,4^{\circ}$ & 0,103 & 4,569 & $33,3^{\circ}$ & 1,330 & 0,115 \\
\hline $\begin{array}{l}\text { AL-HUSSAINI } \\
\text { B }\end{array}$ & -1924 & -800 & -483 & -0.43 & 0,47 & $-0,578$ & 0,036 & $27,4^{\circ}$ & 0,060 & 3,983 & $33,3^{\circ}$ & 1,160 & 0,060 \\
\hline
\end{tabular}

Tableau 2 Etude de la dilatance du sol en écrouissage 


\subsubsection{Direction donnée par la théorie de Rowe}

Rowe indique la relation :

$$
\text { avec } \begin{aligned}
R & =K D \\
R & =\frac{\sigma_{1}}{\sigma_{3}} \\
K=\frac{1+\sin \phi_{c v}}{1-\sin \phi_{c v}} & \text { en déformation plane } \\
D & =\frac{\varepsilon_{3}^{p}}{\varepsilon_{1}^{p}}
\end{aligned}
$$

la relation (12) se met sous la forme:

$$
\mathrm{D}=\frac{\mathrm{R}}{\mathrm{K}} \text {. }
$$

La connaissance des contraintes principales majeures et mineures et de l'angle $\phi_{\mathrm{ov}}$ (colonne 10 du tableau 1) permet de calculer le terme de dilatance $D$ de Rowe. D'autre part, la relation (5) se transforme en :

$$
\operatorname{tg} \gamma^{\prime}=\frac{D \sqrt{3}}{2+D}
$$

ce qui permet de trouver les valeurs $\gamma^{\prime}$ déduites de la théorie de Rowe (colonne 13 du tableau 1). On constate un écart moyen de $1,9^{\circ}$ par rapport au $\gamma^{\prime}$ expérimental.

\subsubsection{Direction donnée par notre théorie}

La valeur de l'angle $\gamma^{\prime}$ est donnée par la formule (6) qui a été démontrée précédemment. II est nécessaire de connaître langle de frottement intergranulaire $\phi_{\mu}$ (colonne 9 du tableau 1) pour trouver $\gamma^{\prime}$ théorique (colonne 11 du tableau 1). On constate un écart moyen de $1,1^{\circ}$ par rapport au $\gamma^{\prime}$ expérimental, ce qui est au moins aussi bon que les résultats donnés par la théorie de Rowe.

\subsection{2 Étude de la dilatance}

Nous étudierons la dilatance sous la forme du rapport :

$$
\frac{\varepsilon_{\text {oct }}^{p}}{\varepsilon_{\text {oct }}^{\prime p}}
$$

utilisé dans notre théorie.

\subsubsection{Valeur de la dilatance expérimentale}

La connaissance des déformations plastiques principales expérimentales (colonnes 4 et 5 du tableau 2) permet de calculer le terme de dilatance tel qu'il est défini par la formule (18) (colonne 7 du tableau 2).

\subsubsection{Valeur de la dilatance donnée par la théorie de Rowe}

La dilatance $\mathrm{D}$ de Rowe est donnée par la relation (16). Du fait que l'on est en déformation plane, on a $\varepsilon_{2}^{p}=0$. On en déduit alors:

$$
\varepsilon_{\text {oct }}^{p}=\frac{\varepsilon_{1}^{P}}{3}(1-D)
$$

de même $\varepsilon_{o c t}^{\prime p}=-\frac{\varepsilon_{T}^{p}}{3} \sqrt{(1+D)^{2}+1+D^{2}}$.

D'où l'on tire :

$$
\frac{\varepsilon_{\text {oct }}^{p}}{\varepsilon_{\text {oct }}^{\prime p}}=\frac{D-1}{\sqrt{(1+D)^{2}+1+D^{2}}}
$$

Cette formule exprime la dilatance déduite de la théorie de Rowe. Connaissant les contraintes principales et l'angle de frottement $\phi_{\mathrm{cv}}$ on en déduit les valeurs portées en colonne 13 du tableau 2).

Les résultats théoriques sont du même ordre de grandeur que les résultats expérimentaux. L'analyse statistique indique un coefficient de corrélation de 0,942 proche de l'unité.

\subsubsection{Valeur de la dilatance donnée par notre théorie}

La relation (2) est aussi valable en déformation plastique totale. Elle peut se mettre sous la forme :

$$
\frac{\varepsilon_{\text {oct }}^{p}}{\varepsilon_{\text {oct }}^{\prime p}}=-\frac{\tau_{\text {oct }}}{\sigma_{\text {oct }}}-\operatorname{tg} \phi_{\mu}
$$

ce qui permet connaissant les contraintes principales et l'angle de frottement $\phi_{4}$ de trouver la dilatance théorique (colonne 9 du tableau 2). On constate là encore, que les résultats sont du même ordre de grandeur que les valeurs expérimentales. L'analyse statistique indique un coefficient de corrélation de 0,992 donc légèrement meilleur que le coefficient trouvé avec la théorie de Rowe.

D'autre part, pour trois essais, il a été possible d'étudier l'évolution de la dilatance expérimentale en fonction du frottement moyen pendant la phase d'écrouissage. Cette étude est faite sur les figures 1 à 3. La formule (20) montre que théoriquement, on doit trouver une droite de pente -1 . Les droites de régression qui ont été tracées ont des pentes comprises entre $-0,78$ et $-1,35$ et des coefficients de corrélation variant de 0,937 à 0,963 .

\section{3 Étude du comportement du sol au pic de cisaillement}

A l'état ultime, nous avons posé comme hypothèse que les déformations plastiques sont de nature différentes. La déformation plastique expérimentale sera considérée comme la variation de déformation se produisant quand le sol se trouve au maximum du cisaillement (colonnes 4 et 5 des tableaux 3 et 4 ).

\subsection{1 Étude de la direction de la distorsion plastique $d \varepsilon_{o c t}^{\prime p}$ dans le plan octaédrique}

\subsubsection{Direction expérimentale}

La direction de la distorsion plastique d $\varepsilon_{0 c t}^{\prime p}, \gamma^{\prime}$ est donnée par la formule (5). Les résultats sont portés en colonne 7 du tableau 3. La direction du cisaillement octaédrique est donnée par l'angle $\gamma$ déduit de la formule (4). On constate un écart moyen de $18,7^{\circ}$ proche de l'écart observé au cours de l'écrouissage.

\subsubsection{Direction donnée par la théorie de Rowe}

Cette direction est donnée par la relation (17) connaissant les contraintes et l'angle $\phi_{\mathrm{cv}}$. Les valeurs de $\gamma^{\prime}$ sont portées en colonne 13 du tableau 3 ).

On observe un écart moyen nul entre les valeurs expérimentales et les valeurs théoriques.

\subsubsection{Direction donnée par notre théorie}

La direction $\gamma^{\prime}$ de la distorsion plastique est donnée par 


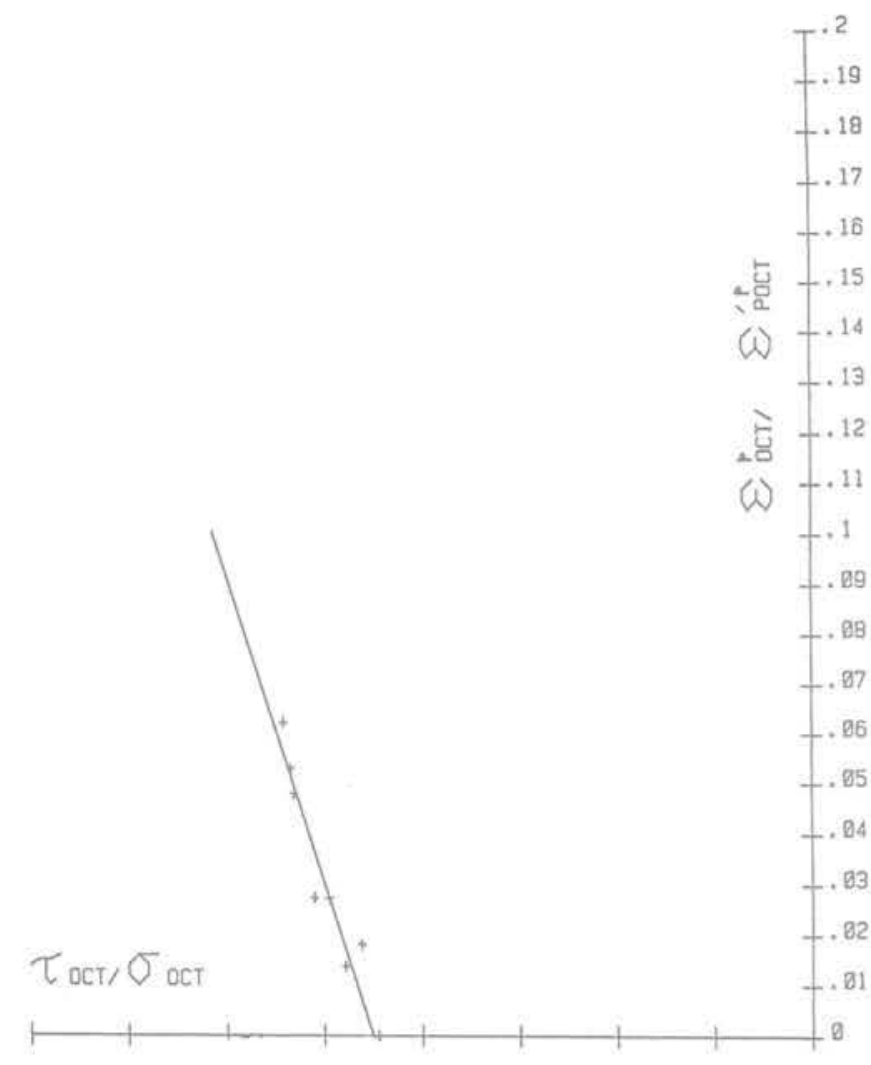

i i i

Fig. 1 Etude de la dilatance pendant l'écrouissage pour l'essai A de Al Hussaini

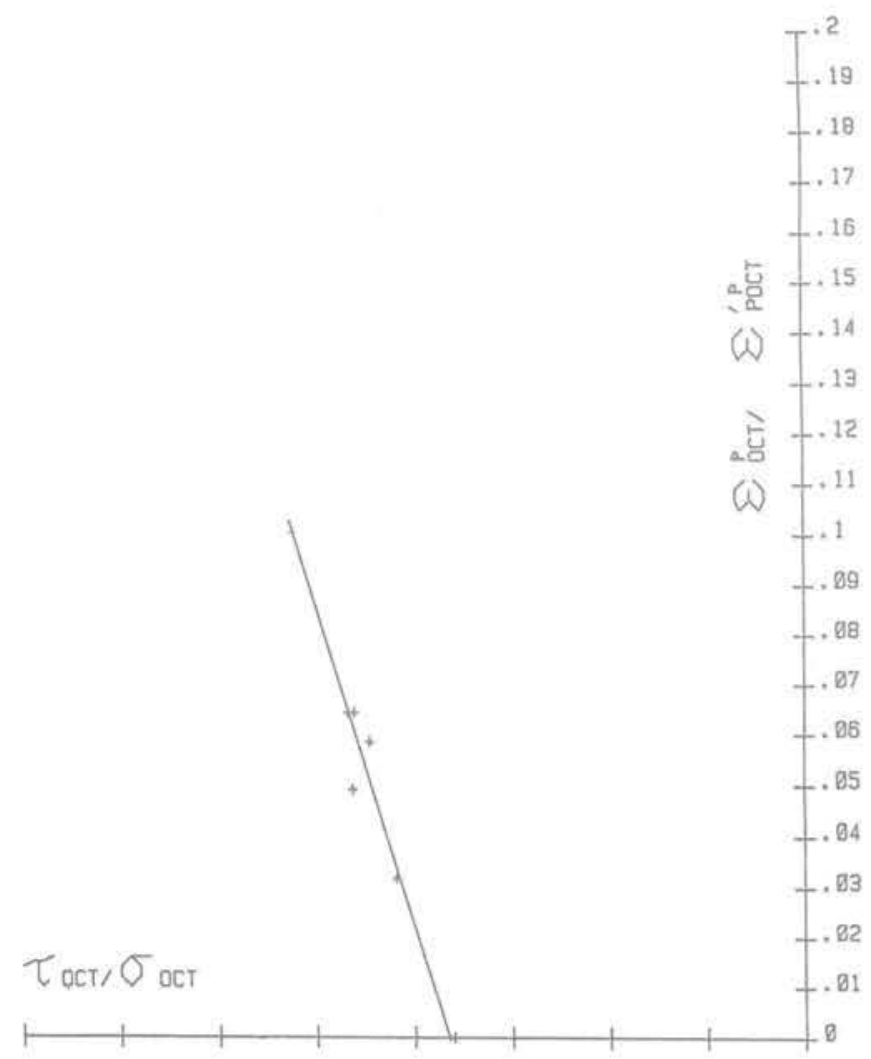

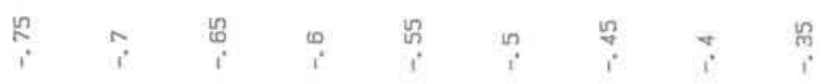

Fig. 3 Etude de la dilatance pendant l'écrouissage pour I'essai CT1 - L2 de Lade

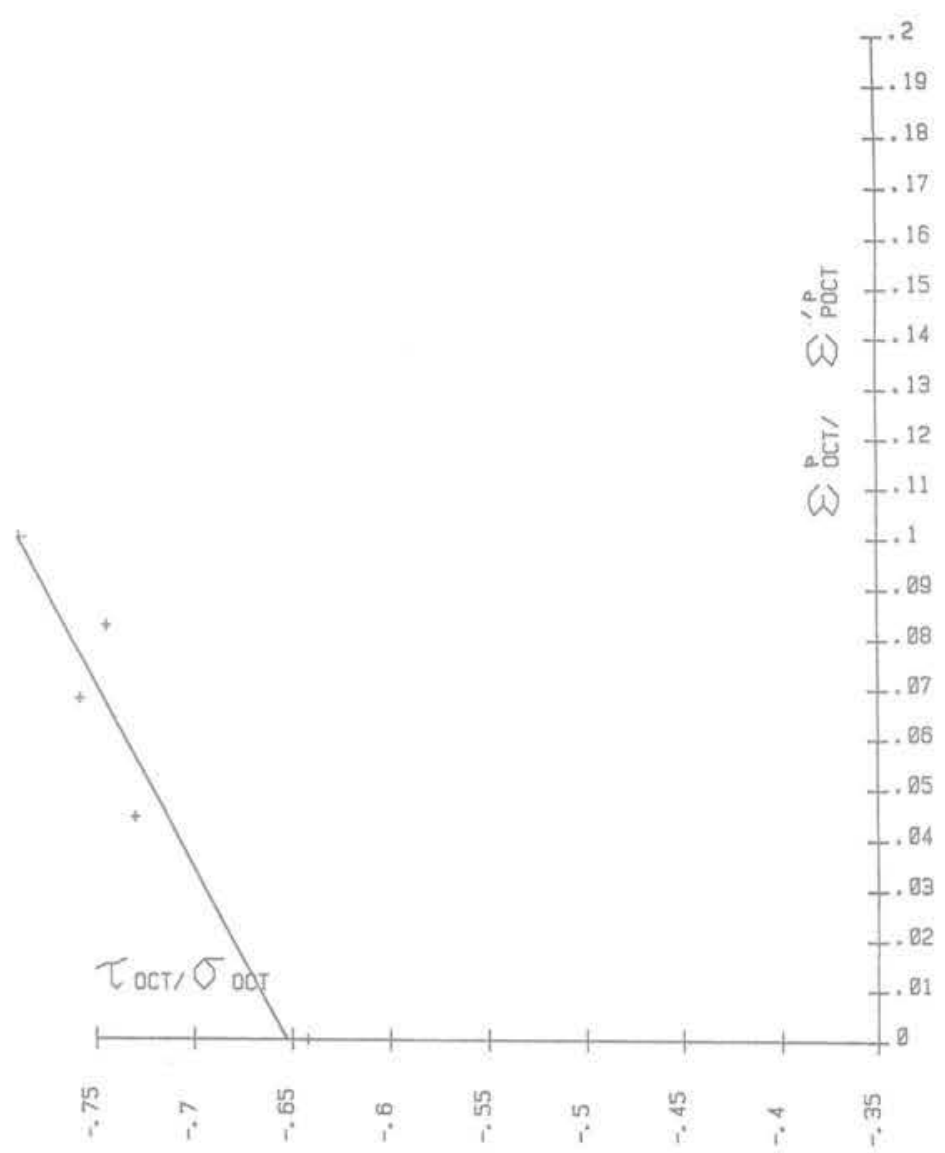

Fig. 2 Etude de la dilatance pendant l'écrouissage pour l'essai CT1 - D2 de Lade 


\begin{tabular}{|c|c|c|c|c|c|c|c|c|c|c|c|c|c|}
\hline Essai & \begin{tabular}{cc|}
$\sigma_{1}$ & \\
$\mathrm{kPa}$ & \\
& \\
& 1
\end{tabular} & \begin{tabular}{cc|}
$\sigma_{2}$ & \\
$\mathrm{kPa}$ & \\
& 2
\end{tabular} & 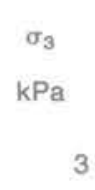 & $\begin{array}{ll}\mathrm{d} \varepsilon_{1}^{\circ} & \\
\% & \\
& 4\end{array}$ & \begin{tabular}{l|l}
$d \varepsilon^{p}$ & \\
$\%$ & \\
& 5
\end{tabular} & $\begin{array}{c}\begin{array}{c}\gamma \\
\text { degré } \\
6\end{array} \\
6\end{array}$ & $\begin{array}{c}\gamma^{\prime} \\
\text { degré } \\
7 \\
7\end{array}$ & $\frac{\tau_{\text {oct }}}{\sigma_{\text {oct }}}$ & $\begin{array}{c}\phi_{\mu} \\
\text { degré } \\
\text { exp. } \\
9\end{array}$ & \begin{tabular}{l}
\multicolumn{1}{c}{$\phi_{\mathrm{cv}}$} \\
degré \\
10
\end{tabular} & $\begin{array}{c}\gamma^{\prime} \\
\text { théorique } \\
\text { degré } \\
\\
11\end{array}$ & 12 & $\begin{array}{r}\gamma^{\prime} \\
\text { Rowe } \\
\text { degré } \\
13\end{array}$ \\
\hline $\begin{array}{l}\text { LADE } \\
\text { CT1-L2 }\end{array}$ & $-313,8$ & $-162,3$ & -60 & $-1,067$ & 1,249 & $-156,4^{\circ}$ & $-147,4^{\circ}$ & $-0,583$ & 26,4 & 41,3 & $-146,5^{\circ}$ & 5,230 & $-148.9^{\circ}$ \\
\hline $\begin{array}{l}\text { LADE } \\
\text { CT1-D2 }\end{array}$ & -703 & -280 & -60 & $-0,277$ & 0,602 & $-160,3^{\circ}$ & $-138,0^{\circ}$ & $-0,767$ & 26,4 & 41,3 & $-139,0^{\circ}$ & 11,717 & $-136.6^{\circ}$ \\
\hline CORNFORTH & -1154 & -493 & -276 & $-0,50$ & 0,61 & $-166,3^{\circ}$ & $-146,7^{\circ}$ & $-0,583$ & 26,6 & 33,2 & $-146,7^{\circ}$ & 4,181 & $-146,7^{\circ}$ \\
\hline $\begin{array}{l}\text { AL-HUSSAINI } \\
\text { A }\end{array}$ & -2207 & -876 & -483 & $-0,860$ & 1,160 & $-167,4^{\circ}$ & $-145,1^{\circ}$ & $-0,621$ & 27,4 & 33,3 & $-145,8^{\circ}$ & 4,569 & $-145,3^{\circ}$ \\
\hline $\begin{array}{l}\text { AL-HUSSAINI } \\
\text { B }\end{array}$ & -1924 & -800 & -483 & $-1,30$ & 1,42 & $-167,9^{\circ}$ & $-148.5^{\circ}$ & $-0,578$ & 27,4 & 33,3 & $-147,6^{\circ}$ & 3,983 & $-147,6^{\circ}$ \\
\hline $\begin{array}{l}\text { AL-HUSSAINI } \\
\mathrm{C}\end{array}$ & -1724 & -745 & -483 & $-0,860$ & 0,930 & $-168,4^{\circ}$ & $-148.7^{\circ}$ & $-0,543$ & 27,4 & 33,3 & $-149,0^{\circ}$ & 3,569 & $-149,4^{\circ}$ \\
\hline
\end{tabular}

Tableau 3 Etude de la position du vecteur distorsion plastique et du cisaillement dans le plan octaédrique à l'équilibre limite

\begin{tabular}{|c|c|c|c|c|c|c|c|c|c|c|c|c|c|}
\hline Essai & 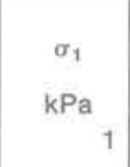 & $\begin{array}{l}\sigma_{2} \\
\mathrm{kPa}\end{array}$ & $\begin{array}{l}\sigma_{3} \\
\mathrm{kPa}_{3}\end{array}$ & $\begin{array}{ll}\mathrm{de}_{1}^{p} & \\
\% & \\
& 4\end{array}$ & $\begin{array}{ll}\mathrm{d} \varepsilon_{3} & \\
\% & \\
& 5\end{array}$ & $\begin{array}{l}\frac{\tau_{\text {oct }}}{\sigma_{\text {oct }}} \\
6 \\
6\end{array}$ & $\begin{array}{l}\frac{d \varepsilon_{\text {oct }}^{p}}{d \varepsilon^{\prime p}{ }_{\text {ot }}} \\
\text { exp. } \\
\quad 7\end{array}$ & \begin{tabular}{r}
\multicolumn{1}{c}{$\phi_{\mu}$} \\
degré \\
8
\end{tabular} & $\begin{array}{r}\frac{d \varepsilon_{\text {ott }}^{p}}{d \varepsilon_{\text {oot }}^{\prime p}} \\
\text { théorique } \\
9\end{array}$ & 10 & $\begin{array}{c}\phi_{c v} \\
\text { degré } \\
11\end{array}$ & $\begin{array}{l}\text { D } \\
12\end{array}$ & $\begin{array}{r}\frac{d \varepsilon_{o t t}^{p}}{d \varepsilon_{\text {oct }}^{\prime p}} \\
\text { Rowe } \\
13\end{array}$ \\
\hline $\begin{array}{l}\text { LADE } \\
\text { CT1-L2 }\end{array}$ & $-313,8$ & $-162,3$ & -60 & $-1,067$ & 1,249 & $-0,583$ & 0,064 & $26,4^{\circ}$ & 0,077 & 5,230 & $41,3^{\circ}$ & 1,071 & 0,028 \\
\hline $\begin{array}{l}\text { LADE } \\
\text { CT1-D2 }\end{array}$ & -703 & -280 & -60 & $-0,277$ & 0,602 & $-0,767$ & 0,295 & $26,4^{\circ}$ & 0,271 & 11,717 & $41,3^{\circ}$ & 2,400 & 0,327 \\
\hline CORNFORTH & -1154 & -493 & -276 & -0.50 & 0,61 & $-0,583$ & 0,081 & $26,6^{\circ}$ & 0,082 & 4,181 & $33,2^{\circ}$ & 1,222 & 0,082 \\
\hline $\begin{array}{l}\text { AL-HUSSAINI } \\
\text { A }\end{array}$ & -2207 & -876 & -483 & $-0,860$ & 1,160 & $-0,621$ & 0,121 & $27,4^{\circ}$ & 0,103 & 4,569 & $33,3^{\circ}$ & 1,330 & 0,115 \\
\hline $\begin{array}{l}\text { AL-HUSSAINI } \\
\text { B }\end{array}$ & -1924 & -800 & -483 & -1.30 & 1,42 & -0.578 & 0,036 & $27,4^{\circ}$ & 0,060 & 3,983 & $33,3^{\circ}$ & 1,160 & 0,060 \\
\hline $\begin{array}{l}\text { AL-HUSSAINI } \\
\text { C }\end{array}$ & -1724 & -745 & -483 & $-0,860$ & 0,930 & $-0,543$ & 0,032 & $27,4^{\circ}$ & 0,025 & 3,569 & $33,3^{\circ}$ & 1,039 & 0,016 \\
\hline
\end{tabular}

Tableau 4 Etude de la dilatance du sol à l'état limite 


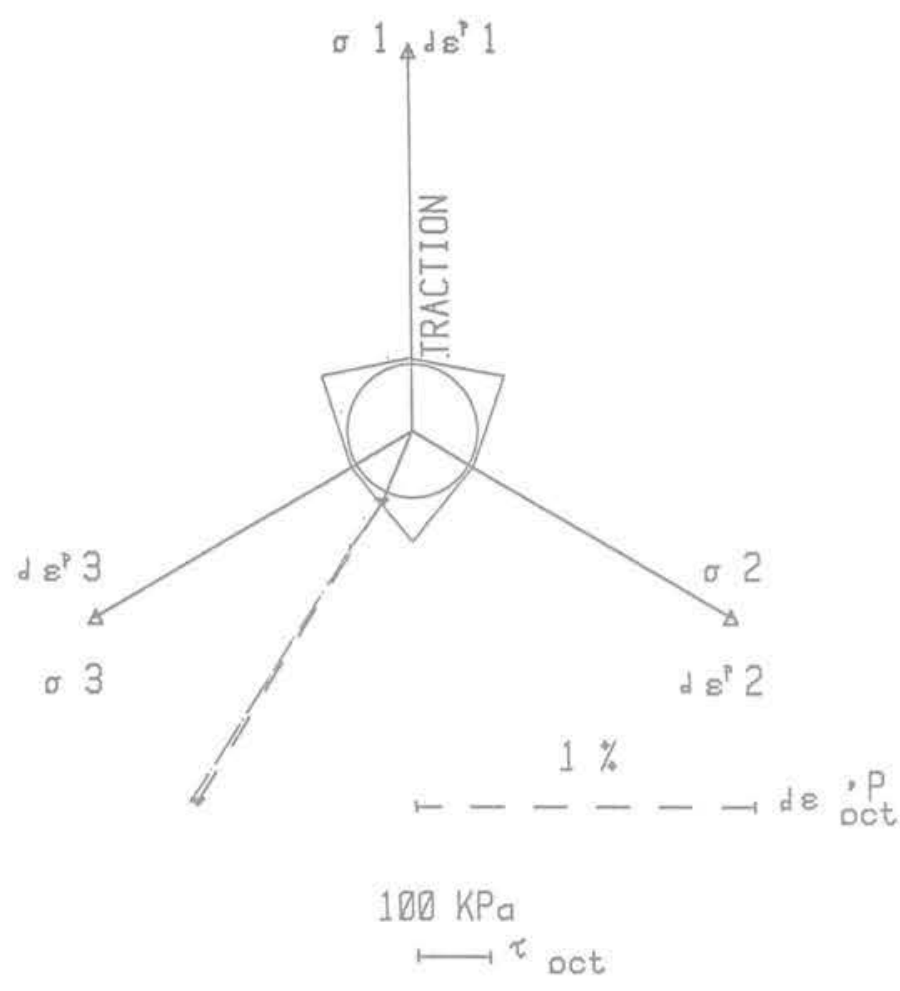

Fig. 4 Etude de la direction plastique dans le plan octaédrique pour I'essai CT1 - L2 de Lade

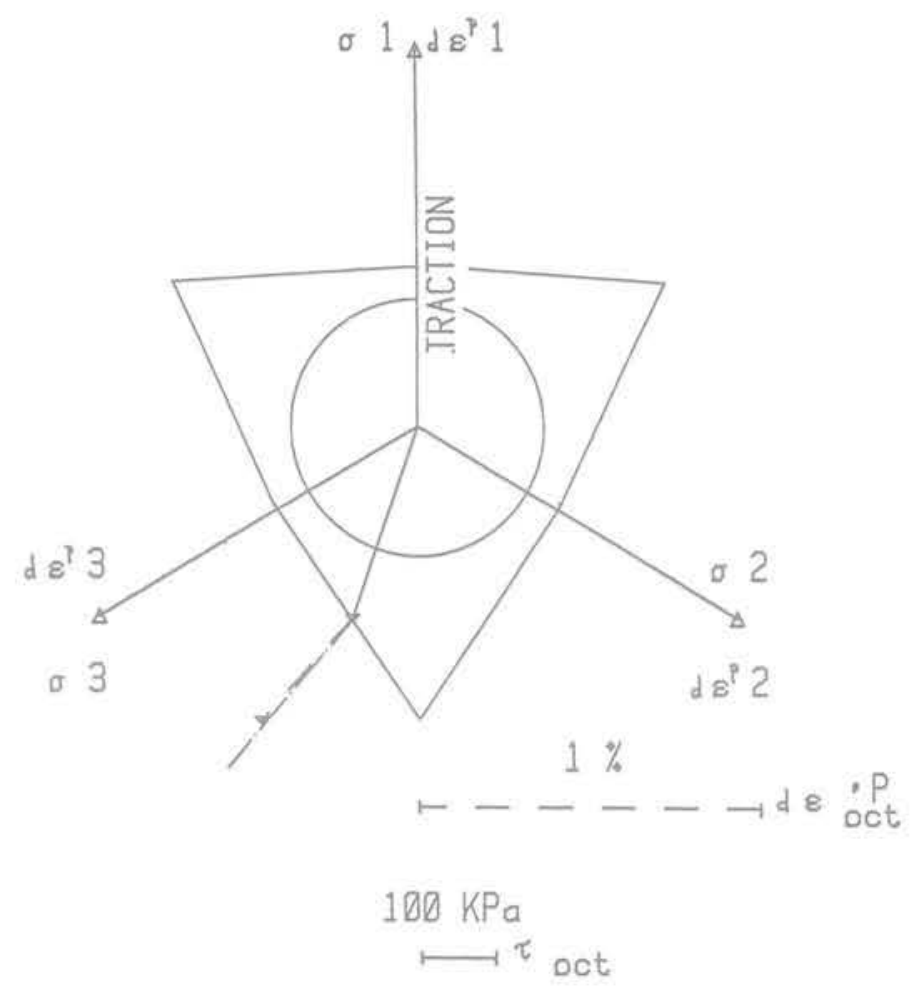

Fig. 5 Etude de la distorsion plastique dans le plan octaédrique pour l'essai CT1 - D2 de Lade

\subsubsection{Dilatance expérimentale}

ration (6), en fonction des contraintes principales et de $\phi_{\mu}$. Les valeurs de $\gamma^{\prime}$ trouvées sont portées en colonne 11 du tableau 3. Là encore, on observe un écart moyen nul entre les valeurs théoriques et les valeurs expérimentales.

Nous avons porté sur les figures 4 et 5 le cisaillement et la distorsion plastique dans le plan octaédrique. Les trois axes disposés à $120^{\circ}$ les uns des autres sont orientés positivement dans le sens de la traction. Le cercle centré sur l'origine correspond au seuil d'écrouissage défini par la relation (1).

Si le point représentatif de l'état de contrainte se trouve à l'intérieur du cercle, on a un comportement élastique. L'hexagone extérieur au cercle correspond à la rupture définie par le critère de Mohr-Coulomb (formule 10). Entre le cercle et l'hexagone, le sol se trouve en écrouissage.

On constate sur les figures 4 et 5 que le point représentant l'état de contrainte est sur l'hexagone correspondant à la rupture. Le vecteur reliant l'origine au point correspond au cisaillement octaédrique. Le vecteur tireté partant de l'hexagone correspond à la distorsion plastique expérimentale. On observe bien un décalage angulaire entre le cisaillement et la distorsion. Le trait d'axe partant de I'hexagone correspond à la direction de la distorsion telle qu'elle est donnée par notre théorie. On observe très bien sur les deux figures, la coincidence entre expérience et théorie.

\subsection{2 Étude de la dilatance à l'état ultime}

Nous étudierons la dilatance sous la forme $d \varepsilon_{\mathrm{oct}}^{p} / \mathrm{d} \varepsilon_{\mathrm{oct}}^{\prime p}$.
La connaissance des déformations plastiques ultimes permet de calculer les valeurs indiquées en colonne 7 du tableau 4.

\subsubsection{Dilatance donnée par la théorie de Rowe}

Cette dilatance est donnée par la formule (19) Connaissant les contraintes et l'angle $\phi_{c v}$, on en déduit les valeurs portées en colonne 13 du tableau 4 qui sont du même ordre de grandeur que les résultats expérimentaux. L'analyse statistique indique un coefficient de corrélation de 0,983 proche de l'unité.

\subsubsection{Dilatance donnée par notre théorie}

Elle est donnée par la relation (2). Connaissant les contraintes et l'angle $\phi_{\mu}$ on en déduit les valeurs portées en colonne 9 du tableau 4. L'analyse statistique indique un coefficient de corrélation de 0,989 légèrement meilleur que celui donné par la théorie de Rowe.

\subsection{Définition de l'angle $\phi_{\mathrm{cv}}$}

Cet angle a été utilisé précédemment. Pour le définir, on part de la relation (12) qui se met sous la forme :

$$
\mathrm{K}=\frac{\mathrm{R}}{\mathrm{D}} \text {. }
$$




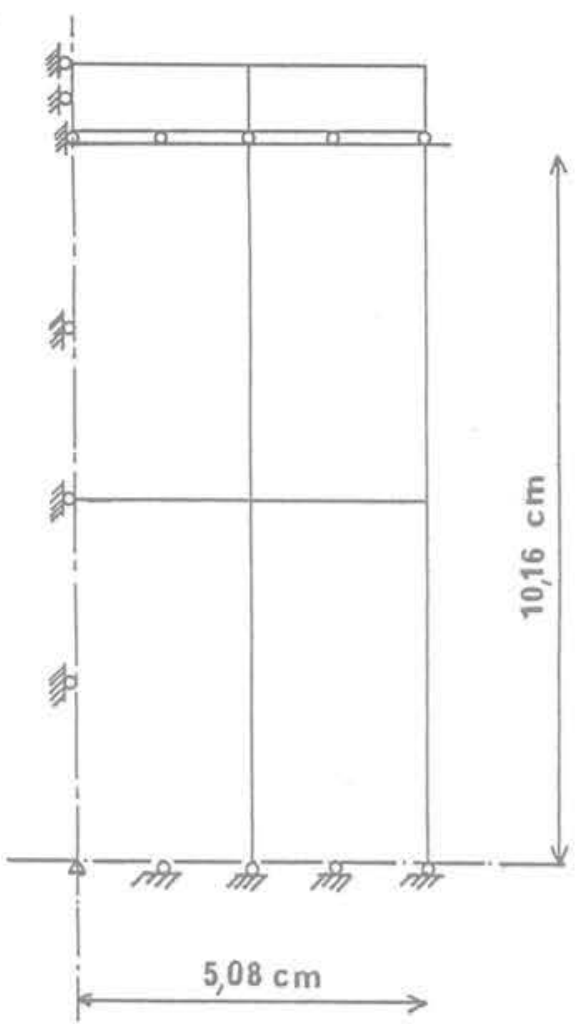

Fig. 6 Le maillage utilisé

La connaissance des contraintes permet de définir la valeur de $\mathrm{A}$ (colonne 10 du tableau 4). A partir des déformations, on trouve le terme $D$ (colonne 12, du tableau 4). On peut donc calculer $\mathrm{K}$ et en déduire $\phi_{\mathrm{ov}}$ par :

$$
\sin \phi_{\mathrm{cv}}=\frac{\mathrm{K}-1}{\mathrm{~K}+1} .
$$

Les valeurs obtenues sont portées en colonne 11. La moyenne des résultats indique $\phi_{\mathrm{cv}}=41,3^{\circ}$ pour le sable de Cornforth, et $\phi_{\mathrm{cv}}=33,3^{\circ}$ pour le sable de Al-Hussaini.

\section{Comparaison de nos calculs théoriques} établis par la méthode des éléments finis et des résultats expérimentaux

\subsection{Maillage}

Le programme Rosalie (Guellec et al 1976) a été modifié pour tenir compte du comportement plastique du sol, mais il n'est pas possible de calculer simultanément le frottement sol-piston et la plasticité dans l'échantillon. Aussi a-t-il été choisi de supprimer le frottement. Les contraintes et les déformations sont alors homogènes et un maillage très simple suffit pour le calcul. Il est présenté sur la figure 6 . On a un rapport hauteur sur largeur de 2 comme pour la majorité des essais. Les éléments utilisés sont des quadrilatères à 8 nceuds. Le long du bord gauche qui représente un plan de symétrie de l'échantillon, les déplacements horizontaux sont nuls, et les déplacements verticaux sont libres. Le long du bord inférieur qui représente le second plan de symétrie, les déplacements verticaux sont nuls et les déplacements horizontaux sont libres. On charge le modèle par l'intermédiaire du piston placé à la partie supérieure.

\subsection{Choix des quatre paramètres constants}

La loi de comportement utilisé demande la définition de 4 paramètres seulement (module de Young, coefficient de Poisson, $\left.\phi_{\mu}, \phi\right)$.

\subsubsection{Définition du coefficient de Poisson}

Au début du chargement, le sol se comporte élastiquement. II faut donc définir à la fois le coefficient de Poisson et le module de Young. Quand on réalise un essai, on a coutume de tracer la courbe de variation de volume en fonction du raccourcissement de l'échantillon. On observe alors que pendant l'état élastique, le sol diminue de volume au chargement, et la courbe est linéaire. On peut mesurer sa pente initiale $p_{1}$, ceci permet de définir le coefficient de Poisson. En effet, les relations de l'élasticité indiquent:

$$
\begin{aligned}
& \varepsilon_{1}=\frac{1}{E}\left(\sigma_{1}-\nu\left(\sigma_{2}+\sigma_{3}\right)\right) \\
& \varepsilon_{2}=\frac{1}{E}\left(\sigma_{2}-\nu\left(\sigma_{1}+\sigma_{3}\right)\right)=0 \\
& \varepsilon_{3}=\frac{1}{E}\left(\sigma_{3}-\nu\left(\sigma_{1}+\sigma_{2}\right)\right) .
\end{aligned}
$$

La seconde relation se transforme en :

$$
\sigma_{2}=\nu\left(\sigma_{1}+\sigma_{3}\right) \text {. }
$$

On trouve également

$$
\frac{\varepsilon_{1}}{\varepsilon_{3}}=\frac{\sigma_{1}-\nu\left(\sigma_{2}+\sigma_{3}\right)}{\sigma_{3}-\nu\left(\sigma_{1}+\sigma_{2}\right)} .
$$

En tenant compte de la relation précédente on peut éliminer $\sigma_{2}$ et en changeant l'origine de mesure des contraintes en posant $\sigma_{3}^{\prime}=0$ et $\sigma_{1}^{\prime}=\sigma_{1}-\sigma_{3}$ on obtient alors :

$$
\frac{\varepsilon_{1}}{\varepsilon_{3}}=-\frac{v}{1-v} \text {. }
$$

Or, la variation de volume élastique du sol en déformation plane s'écrit :

$$
\begin{aligned}
& \frac{\Delta v}{v}=\theta=\varepsilon_{1}+\varepsilon_{3} \\
& y=\frac{\theta}{\varepsilon_{1}}=1+\frac{\varepsilon_{3}}{\varepsilon_{1}} .
\end{aligned}
$$

d'oủ

$p_{1}$ étant la pente initiale de la courbe de variation de volume du sol que l'on peut mesurer facilement. En tenant compte de la formule (22), on trouve alors:

$$
v=\frac{1-p_{1}}{2-p_{1}}
$$

Le tableau 5 indique en colonne 1, la pente initiale de la courbe de variation de volume pour chaque essai. On en déduit alors le coefficient de Poisson indiqué en colonne 2.

\subsubsection{Définition du module de Young}

Les relations de l'élasticité indiquent:

$$
\varepsilon_{1}=\frac{1}{E}\left(\sigma_{1}-v\left(\sigma_{2}+\sigma_{3}\right)\right) \text {. }
$$


Si l'on tient compte de la condition de déformation plane (21), on a :

$$
\varepsilon_{1}=\frac{1}{E}\left[\sigma_{1}\left(1-v^{2}\right)-v \sigma_{3}(1+v)\right] .
$$

On fait maintenant un changement d'origine des pressions tels que $\sigma_{3}$ s'annule. II vient alors:

$$
\begin{aligned}
& p_{2}=\frac{\sigma_{1}-\sigma_{3}}{\varepsilon_{1}}=\frac{E}{1-v^{2}} \\
\text { ou encore } & E=p_{2}\left(1-\nu^{2}\right)
\end{aligned}
$$

où $p_{2}$ est la pente initiale de la courbe déviateur en fonction de l'écrasement de l'échantillon. Cette pente a été portée en colonne 6 du tableau 5 . En tenant compte de $v$ (colonne 2), on obtient par la relation (24), le module de Young (colonne 7).

\subsubsection{Définition de l'angle $\phi$}

L'angle de frottement interne $\phi$ est donné par le critère de Mohr-Coulomb.

3.24 Définition de l'angle $\phi_{\mu}$

La relation (2) peut se mettre sous la forme:

$$
\operatorname{tg} \phi_{\mu}=-\frac{\tau_{\text {oct }}}{\sigma_{\text {oct }}}-\frac{d \varepsilon_{o c t}^{p}}{d \varepsilon_{o c t}^{\prime p}} .
$$

Connaissant les contraintes à l'équilibre limite, on peut calculer le frottement $\frac{\tau_{\text {oct }}}{\sigma_{\text {oct }}}$ (colonne 6 du tableau 4). Partant des déformations plastiques à la rupture (colonnes 4 et 5 ), on peut trouver:

$$
\frac{d \varepsilon_{\text {oct }}^{p}}{d \varepsilon_{\text {oct }}^{\prime p}} .
$$

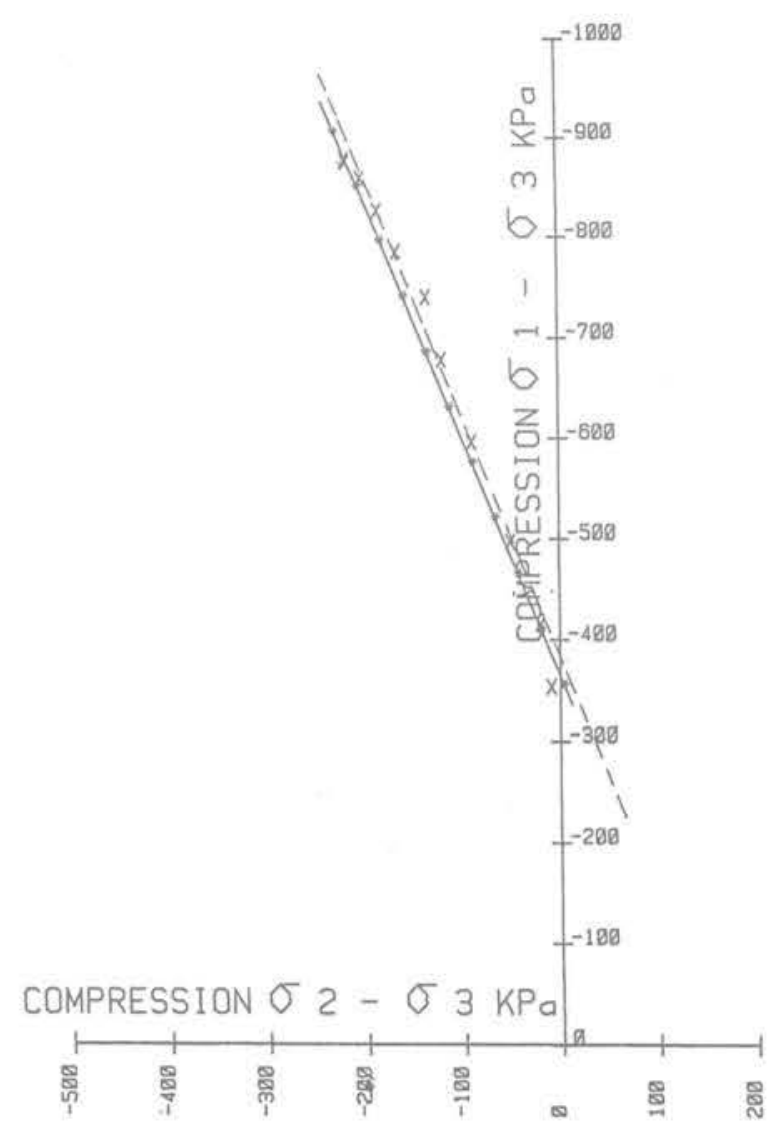

Fig. 7 Etude de la contrainte moyenne calculée et mesurée $\sigma_{2}$ pour l'essai de Cornforth
La relation précédente permet alors de définir tg $\phi_{\mu}$ (colonne 8). La moyenne des valeurs est de $\phi_{\mu}=26,4^{\circ}$ pour le sable de Lade, $\phi_{\mu}=26,6^{\circ}$ pour celui de Cornforth, et $\phi_{\mu}=27,4^{\circ}$ pour Al-Hussaini, ce qui est très proche de $\phi_{\mu}=28^{\circ}$ que Monnet et al. (1979) avaient trouvé pour un sable.

\section{3 Étude de la contrainte intermédiaire $\sigma_{2}$}

Cornforth (1964) considère que la relation élastique (21) est encore valable à la rupture. D'une façon plus générale, on peut généraliser l'hypothèse à l'écrouissage. En procédant à un changement d'origine des pressions, il vient

$$
\nu=\frac{\sigma_{2}-\sigma_{3}}{\sigma_{1}-\sigma_{3}} .
$$

Nous avons donc tracé pour tous les essais, la variation de $\sigma_{2}-\sigma_{3}$ en fonction de $\sigma_{1}-\sigma_{3}$ (figures 7 à 12). Les points expérimentaux sont sensiblement alignés le long de la droite de régression dessinée en tireté. Les coefficients de corrélation sont indiqués en colonne 3 du tableau 5 .

Nous avons également porté sur les figures en trait continu, les résultats donnés par le calcul par éléments finis, pour lesquels la formule 21 est utilisée.

Ces points sont alignés sur des droites de pente correspondant au coefficient de Poisson (colonne $2 \mathrm{du}$ tableau 5). On observe que les droites de régression expérimentales sont proches des droites calculées et ont des pentes voisines (colonne 5 du tableau 5).

On peut donc considérer que la relation 21 est bien valable pendant la plasticité.

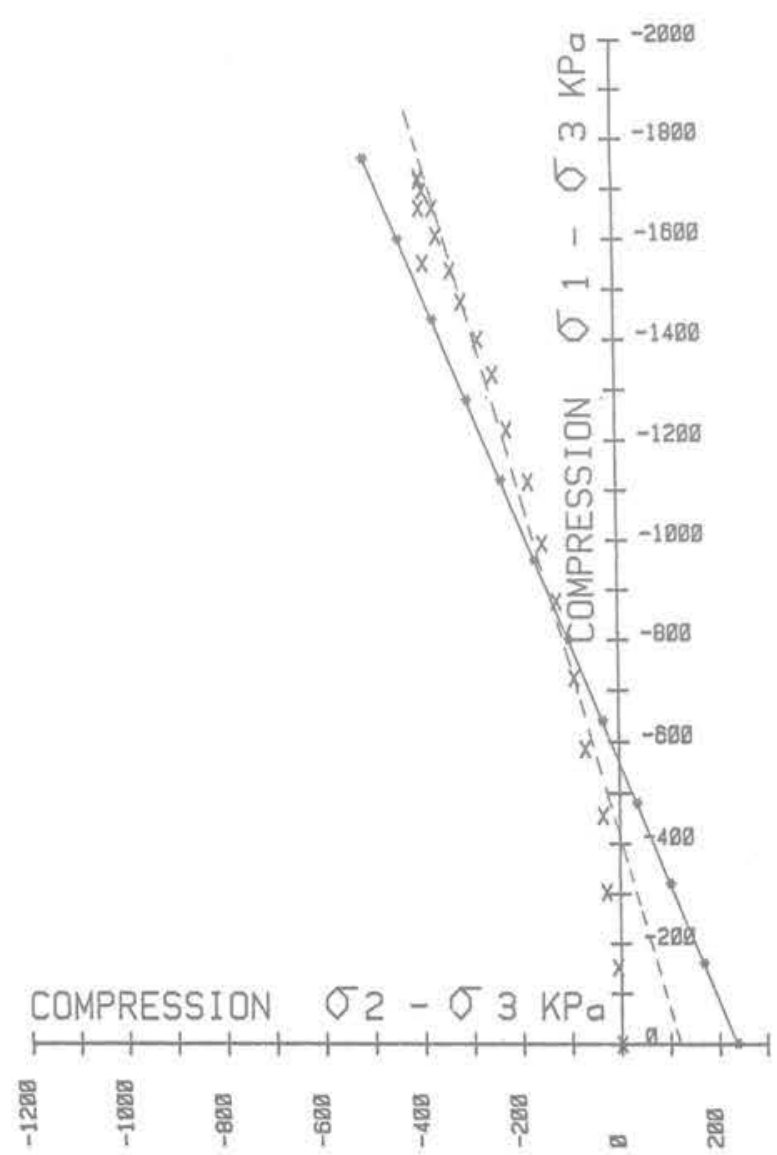

Fig. 8 Etude de la contrainte moyenne calculée et mesurée $\sigma_{2}$ pour l'essai $A$ de Al Hussaini 


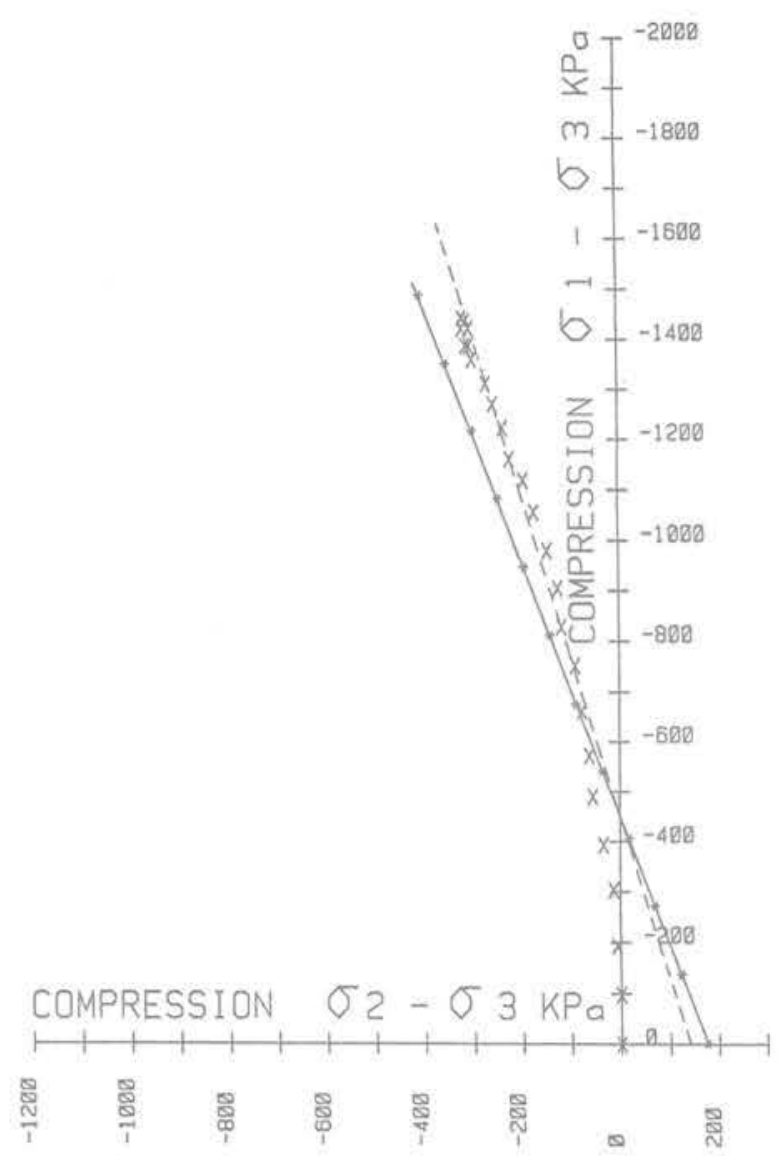

Fig. 9 Etude de la contrainte moyenne calculée et mesurée $\sigma_{2}$ pour l'essai $B$ de Al Hussaini

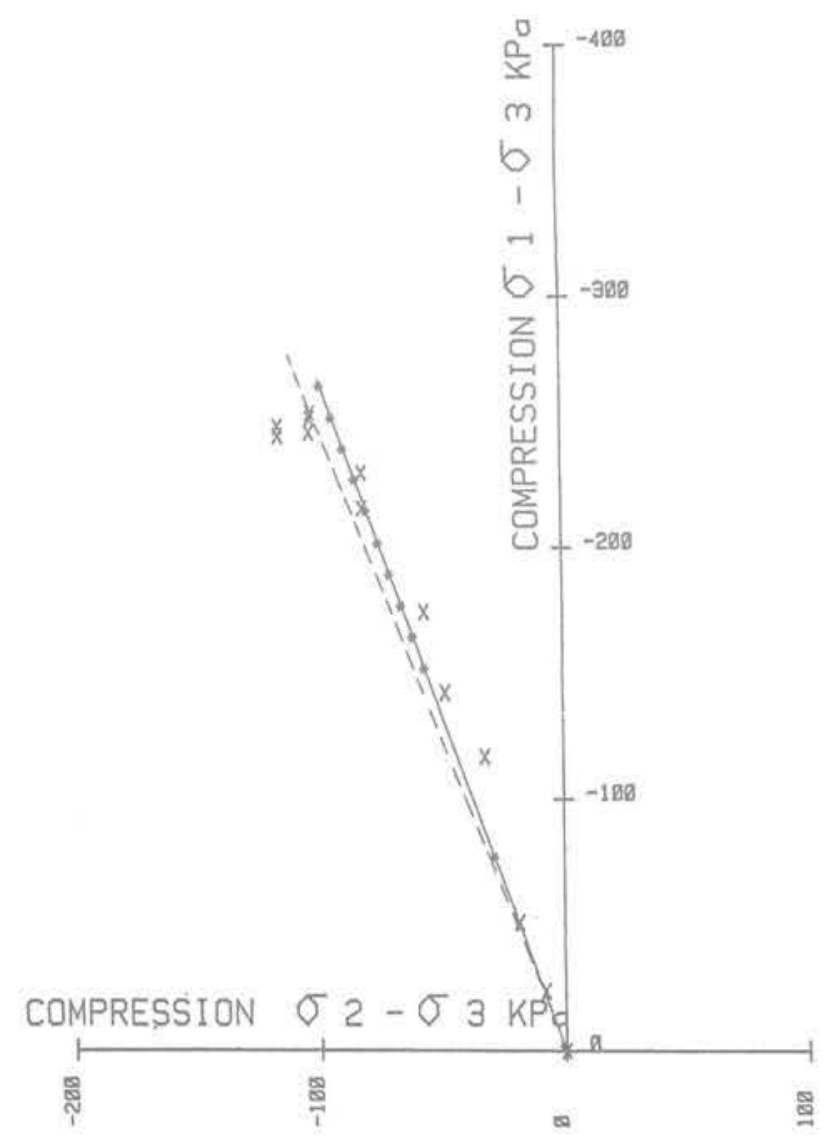

Fig. 11 Etude de la contrainte moyenne calculée et mesurée $\sigma_{2}$ pour I'essai CT1 - L2 de Lade

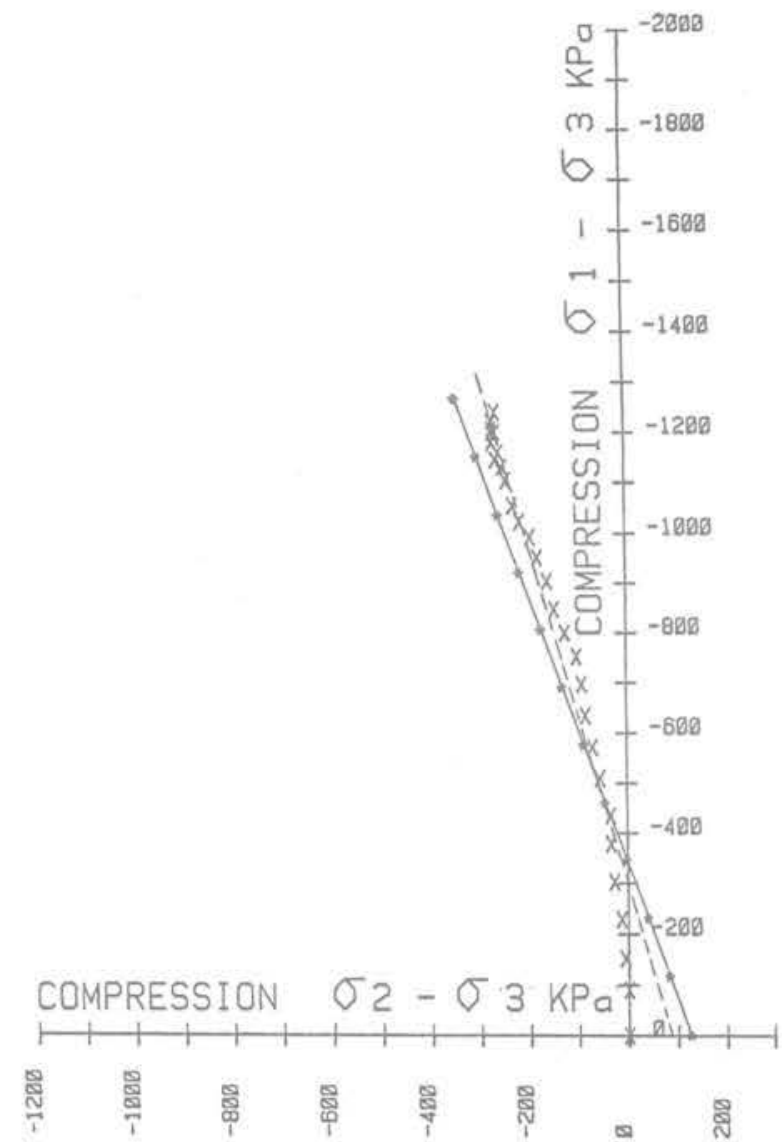

Fig. 10 Etude de la contrainte moyenne calculée et mesurée $\sigma_{2}$ pour l'essai $C$ de Al Hussaini

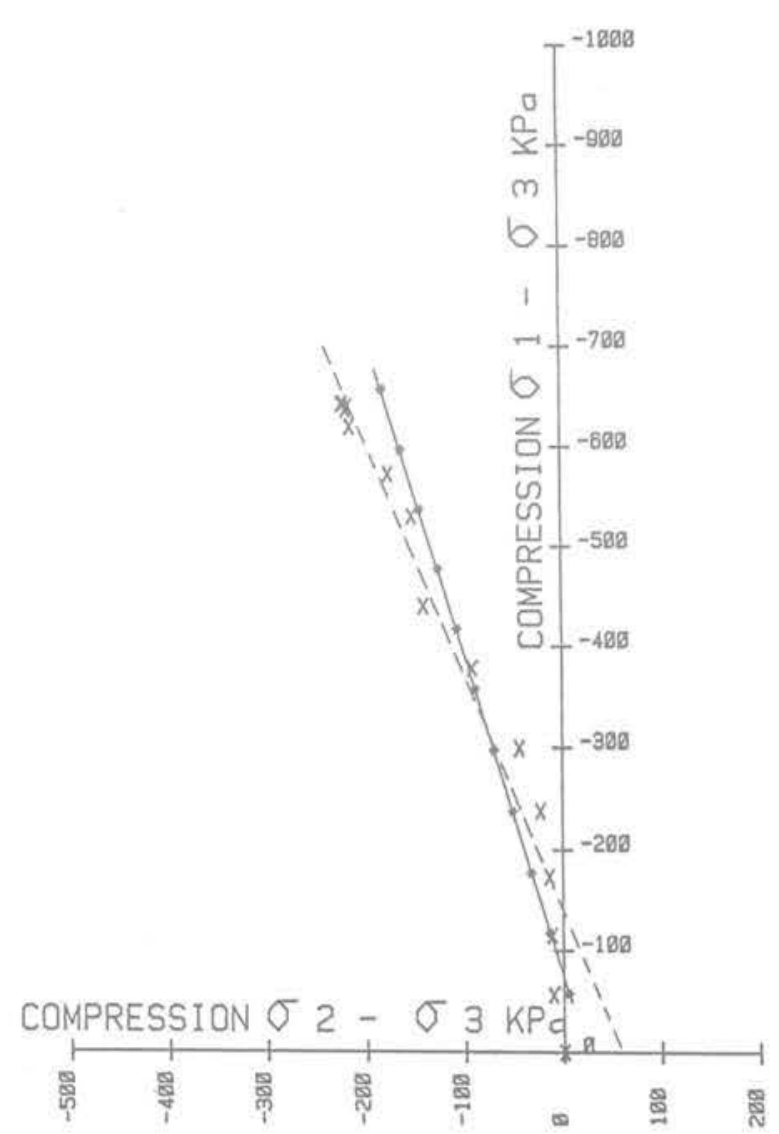

Fig. 12 Etude de la contrainte moyenne calculée et mesurée $\sigma_{2}$ pour l'essai CT1-D2 de Lade 


\begin{tabular}{|c|c|c|c|c|c|c|c|c|c|}
\hline Essai & $p_{1}$ & $\nu$ & $\begin{array}{r}\text { corrélation } \\
3\end{array}$ & $\begin{array}{c}\text { ordonnée à } \\
\text { lorigine } \\
\mathrm{kPa}\end{array}$ & $\mathrm{P}_{3}$ & $\begin{array}{l}\mathrm{p}_{2} \\
\mathrm{kPa}\end{array}$ & $\begin{array}{c}\mathrm{E} \\
\mathrm{kPa}\end{array}$ & $\begin{array}{c}\phi \\
\text { degré } \\
\end{array}$ & $\begin{array}{c}\phi_{\mu} \\
\text { degré }\end{array}$ \\
\hline $\begin{array}{l}\text { LADE } \\
\text { CT1-L2 }\end{array}$ & 0,417 & 0,37 & 0,9835 & 8 & 0,42 & 40980 & 34000 & $42,8^{\circ}$ & $26,4^{\circ}$ \\
\hline $\begin{array}{l}\text { LADE } \\
\text { CT1-D2 }\end{array}$ & 0,550 & 0,31 & 0,9856 & 63,3 & 0,43 & 147000 & 133000 & $57,4^{\circ}$ & $26,4^{\circ}$ \\
\hline CORNFORTH & 0,288 & 0,42 & 0,9945 & 160,8 & 0,42 & 57200 & 47000 & $37,9^{\circ}$ & $26,6^{\circ}$ \\
\hline $\begin{array}{l}\text { AL-HUSSAINI } \\
\text { A }\end{array}$ & 0,274 & 0,42 & 0,9928 & 119,5 & 0,29 & 32770 & 27000 & $39,9^{\circ}$ & $27.4^{\circ}$ \\
\hline $\begin{array}{l}\text { AL-HUSSAINI } \\
\text { B }\end{array}$ & 0.368 & 0,39 & 0,9910 & 141, & 0,31 & 21280 & 18000 & $36,8^{\circ}$ & $27,4^{\circ}$ \\
\hline $\begin{array}{l}\text { AL-HUSSAINI } \\
C\end{array}$ & 0,420 & 0,37 & 0.9846 & 85,3 & 0,29 & 15100 & 13000 & $34,2^{\circ}$ & $27,4^{\circ}$ \\
\hline
\end{tabular}

Tableau 5 Paramètres de la loi de comportement

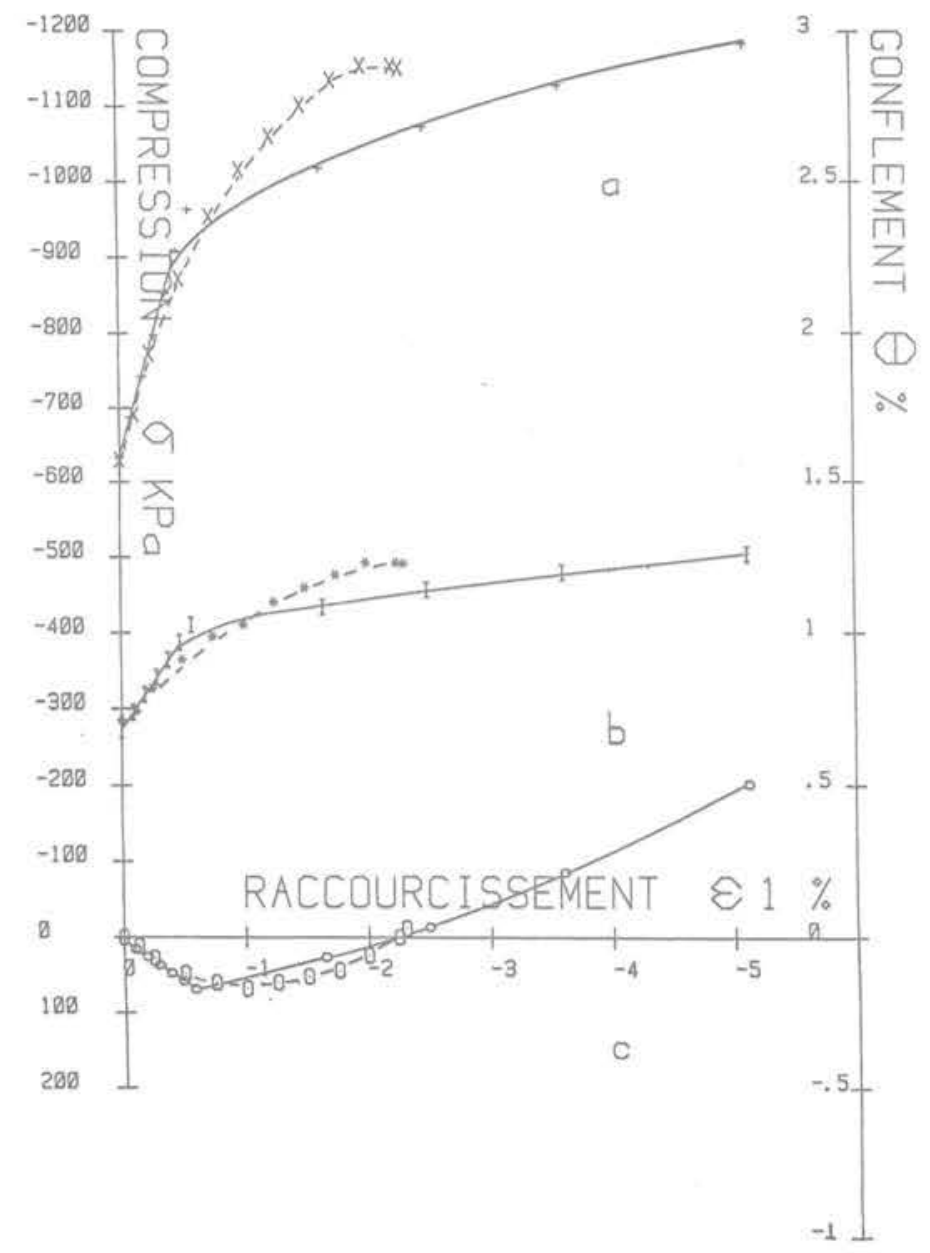

Fig. 13 Comparaison du calcul et de l'expérience pour I'essai de Cornforth, $\sigma_{3}=276 \mathrm{KPa}$

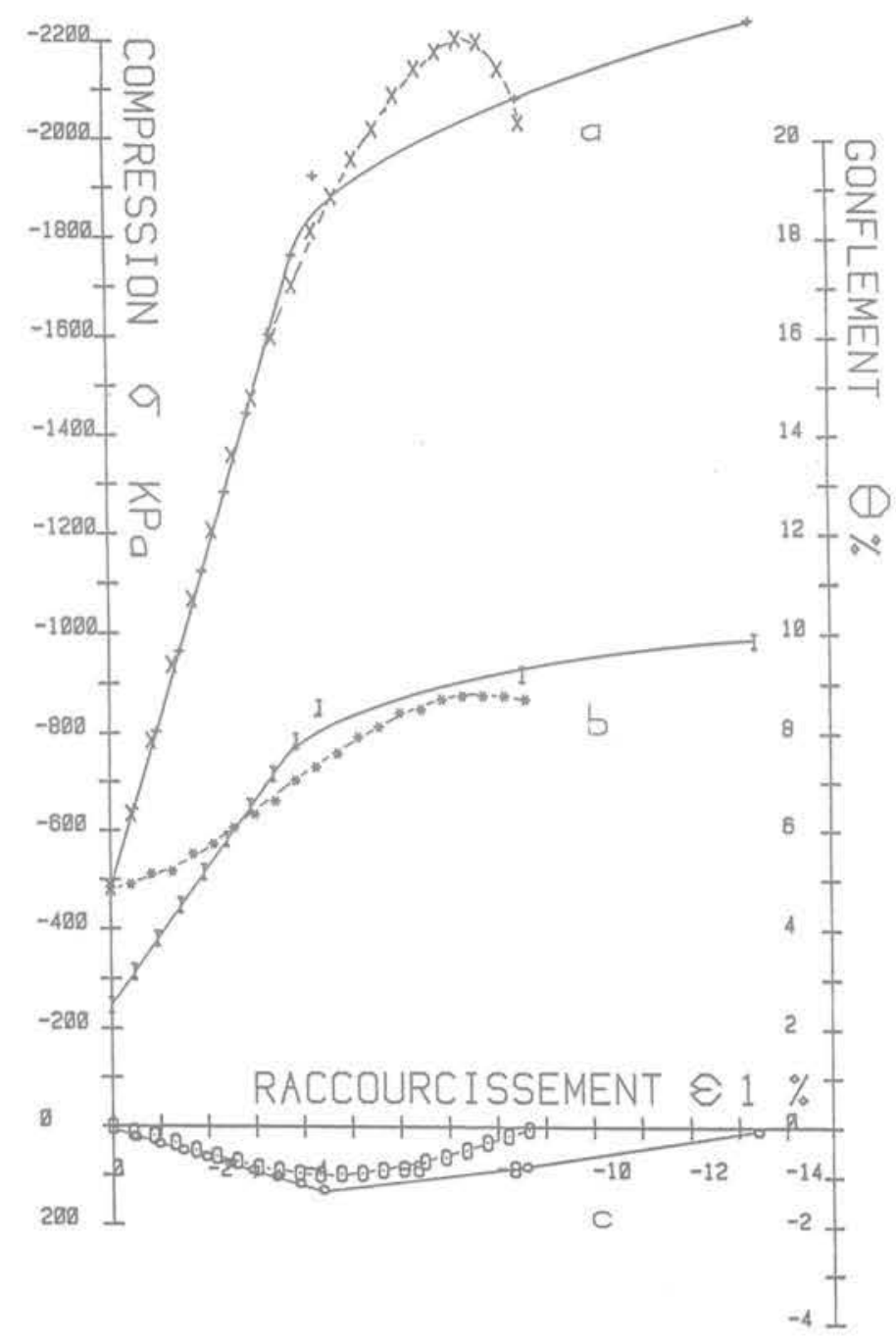

Fig. 14 Comparaison du calcul et de l'expérience pour l'essai A de Al Hussaini, $\sigma_{3}=483 \mathrm{KPa}$ 


\subsection{Calcul de l'essai de Cornforth}

La comparaison des résultats expérimentaux et théoriques a été faite sur la figure 13. Les courbes en pointillés correspondent à l'expérience et les courbes continues au calcul. Les courbes "a montrent la variation de la contrainte principale majeure. Les courbes " $b$ " donnent la contrainte moyenne. Les points " $\mathrm{C}$ montrent la variation de volume du sol. Dans le domaine élastique, on observe une bonne coïncidence, que ce soit en contrainte ou en variation de volume. Par contre, dès qu'on arrive en écrouissage, les déformations calculées sont plus fortes que ce qui est donné par l'expérience.

La rupture, qui correspond au dernier point calculé est obtenue pour un raccourcissement de $5,1 \%$ alors qu'il est de $2,3 \%$ en réalité. Par contre, la courbe de variation de volume est correctement calculée. La différence sur les déplacements peut venir du fait que le sable a un angle de frottement de $37,9^{\circ}$ qui est donc trop fort pour cet échantillon.

L'élancement de 2 de l'éprouvette expérimentale implique en effet que l'angle de frottement soit inférieur à $36,9^{\circ}$.

\subsection{Calcul des essais de Al-Hussaini}

Le sable testé est assez lâche, ce qui fait que le domaine d'écrouissage est faible.

L'étude contrainte-déformation a été faite pour l'essai A sur la figure 14, pour l'essai B sur la figure 15, pour l'essai $\mathrm{C}$ sur la figure 16 . On constate aussi bien en contrainte majeure (courbes " $a n$ ), en contrainte moyenne (courbes " $b$ ) ou en variation de volume (courbes " $\mathrm{C} n$ ) une très bonne coïncidence entre théorie et expérience.

\subsection{Calcul des essais de Lade}

Ces essais sont plus intéressants puisque le sol est dense, ce qui implique des angles de frottement interne élevé $\left(\phi=42,8^{\circ}\right.$ et $\left.57,4^{\circ}\right)$ et donc une zone d'écrouissage étendue. D'autre part, l'échantillon est limité sur toutes ses faces par des plaques, les déformations et les contraintes sont alors parfaitement homogènes et la condition de dimensionnement de l'échantillon n'est plus à respecter. L'étude contrainte-

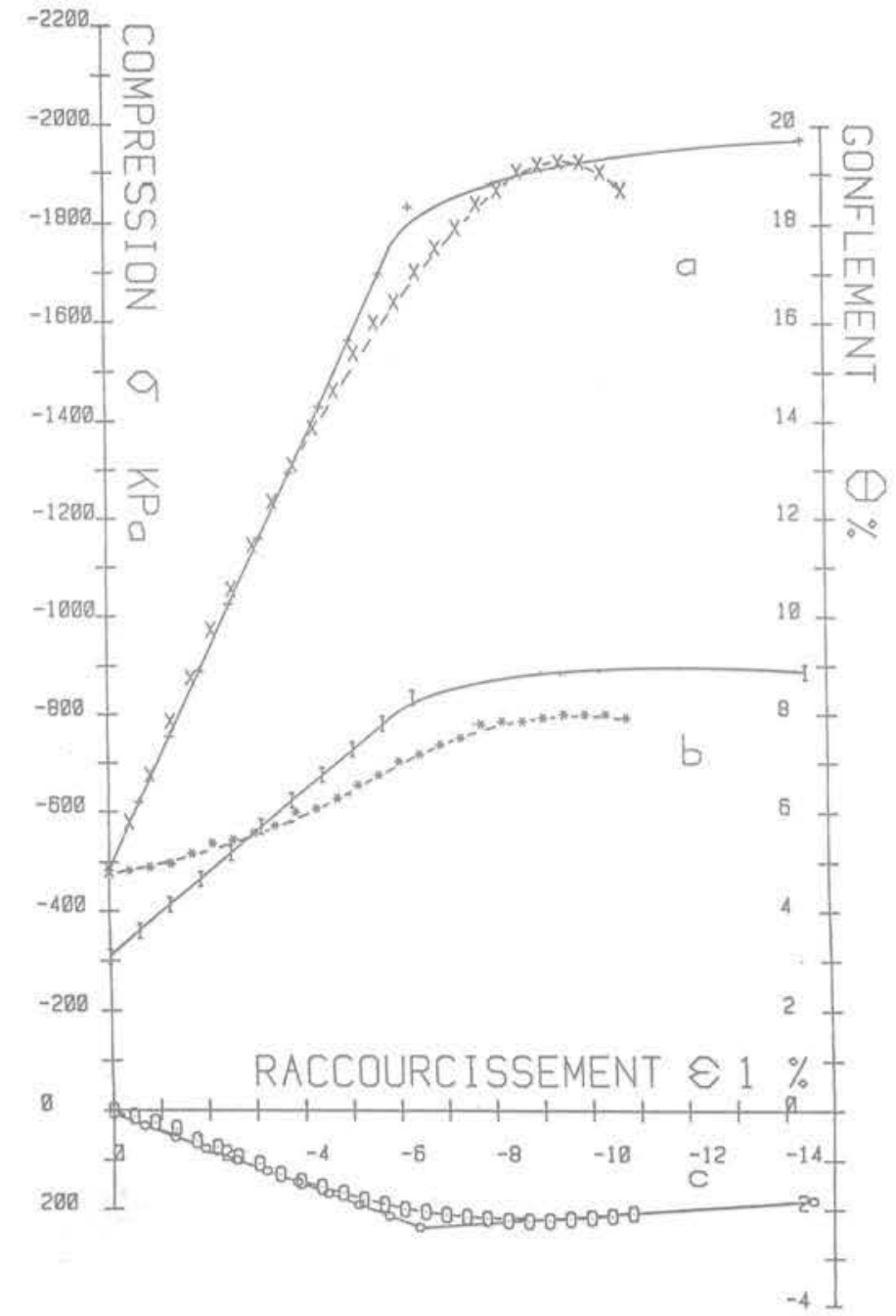

Fig. 15 Comparaison du calcul et de l'expérience pour I'essai $B$ de Al Hussaini, $\sigma_{3}=483 \mathrm{KPa}$

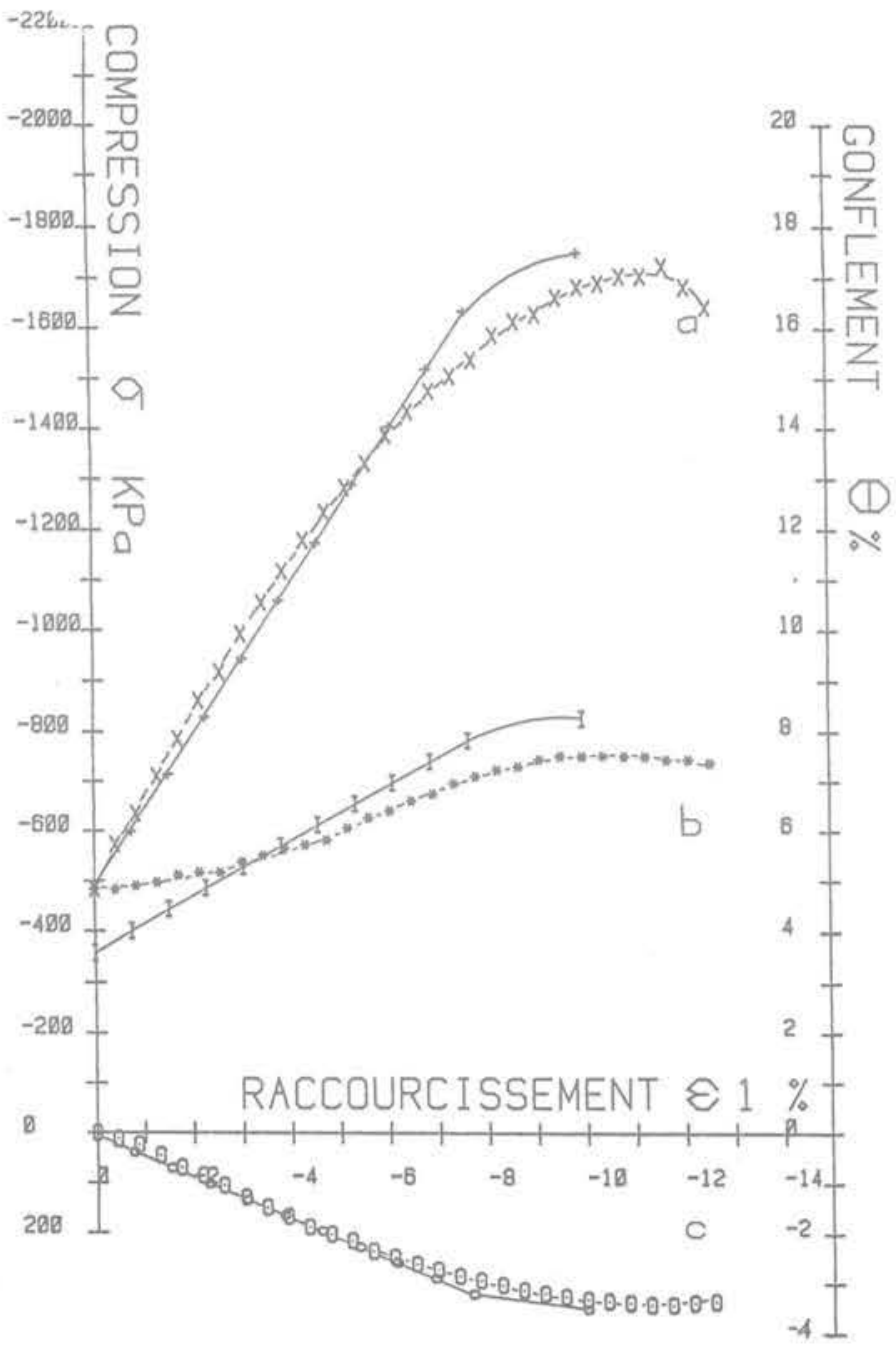

Fig. 16 Comparaison du calcul et de l'expérience pour I'essai $C$ de Al Hussaini, $\sigma_{3}=483 \mathrm{KPa}$ 


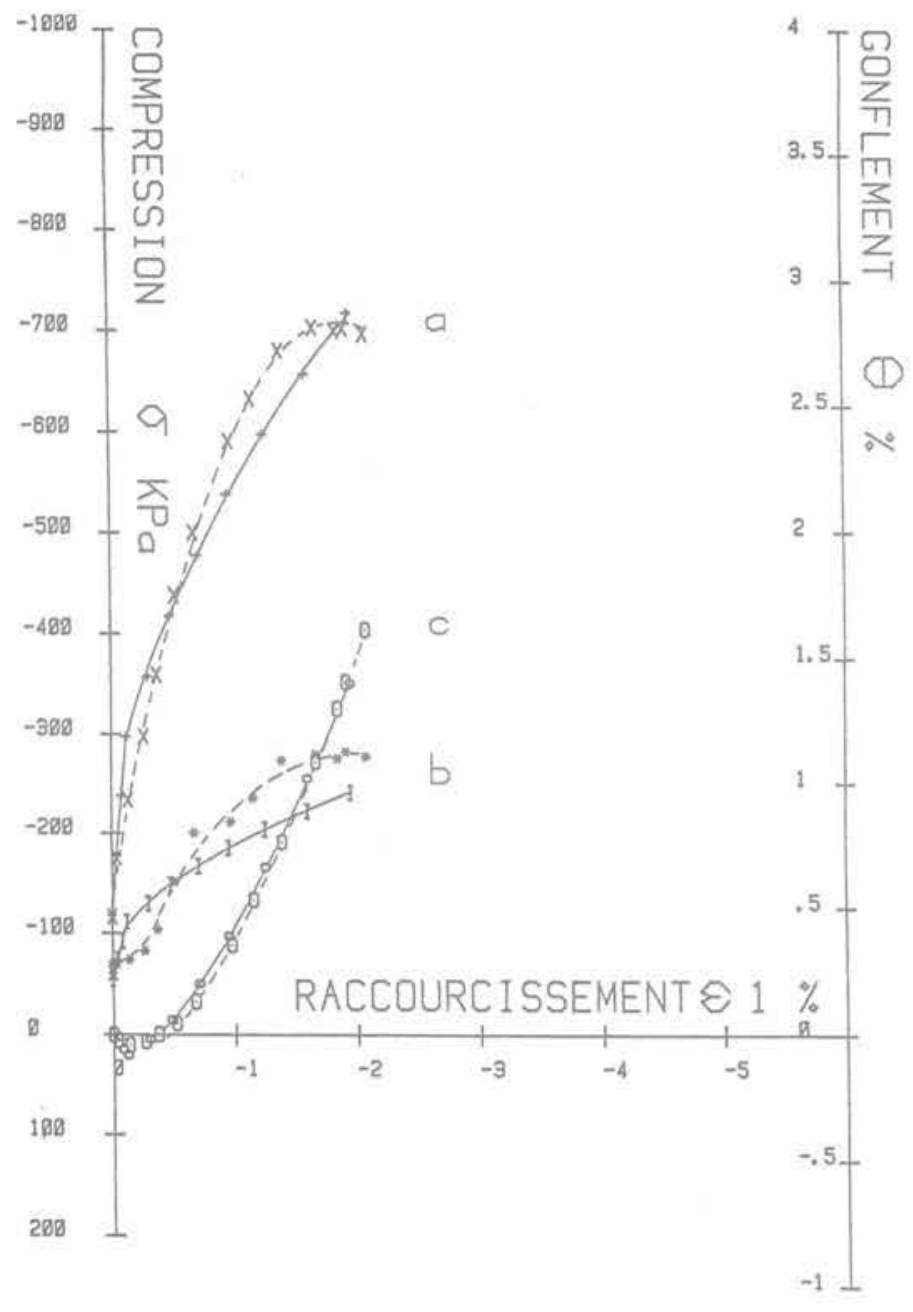

Fig. 17 Comparaison du calcul et de l'expérience pour I'essai CT1 - D2 de Lade, $\sigma_{3}=60 \mathrm{KPa}$

déformation a été faite pour l'essai CT1-D2 sur la figure 17 et pour CT1-L2 sur la figure 18. On observe une coincidence parfaite pour les contraintes maximum et moyennes, mais aussi pour la variation de volume.

\section{Conclusion}

La loi de comportement du sol pulvérulent présentée par Monnet et al. (1979) dans le cas du changement du sol en symétrie axiale a été étendue au cas de la déformation plane.

Moyennant des hypothèses simples concernant la valeur des déformations plastiques d'écrouissage et d'équilibre limite, la théorie permet de retrouver l'expérience aussi bien en ce qui concerne la position de la distorsion plastique dans le plan octaédrique (ou plan moyen), que pour la dilatance. Cette formulation se montre au moins aussi bonne et même parfois meilleure que celle de Rowe.

D'autre part, l'application des formules au calcul par éléments finis permet de retrouver l'expérience pour toute une série d'essais sur des sables denses.

Enfin, cette méthode de calcul est plus simple que la majorité des autres lois de comportement connues, puisqu'elle utilise seulement quatre paramètres constants $\left(E, v, \phi, \phi_{\mu}\right)$. En particulier le paramètre utilisé pour l'écrouissage $\phi_{\mu}$ est le même en symétrie axiale et

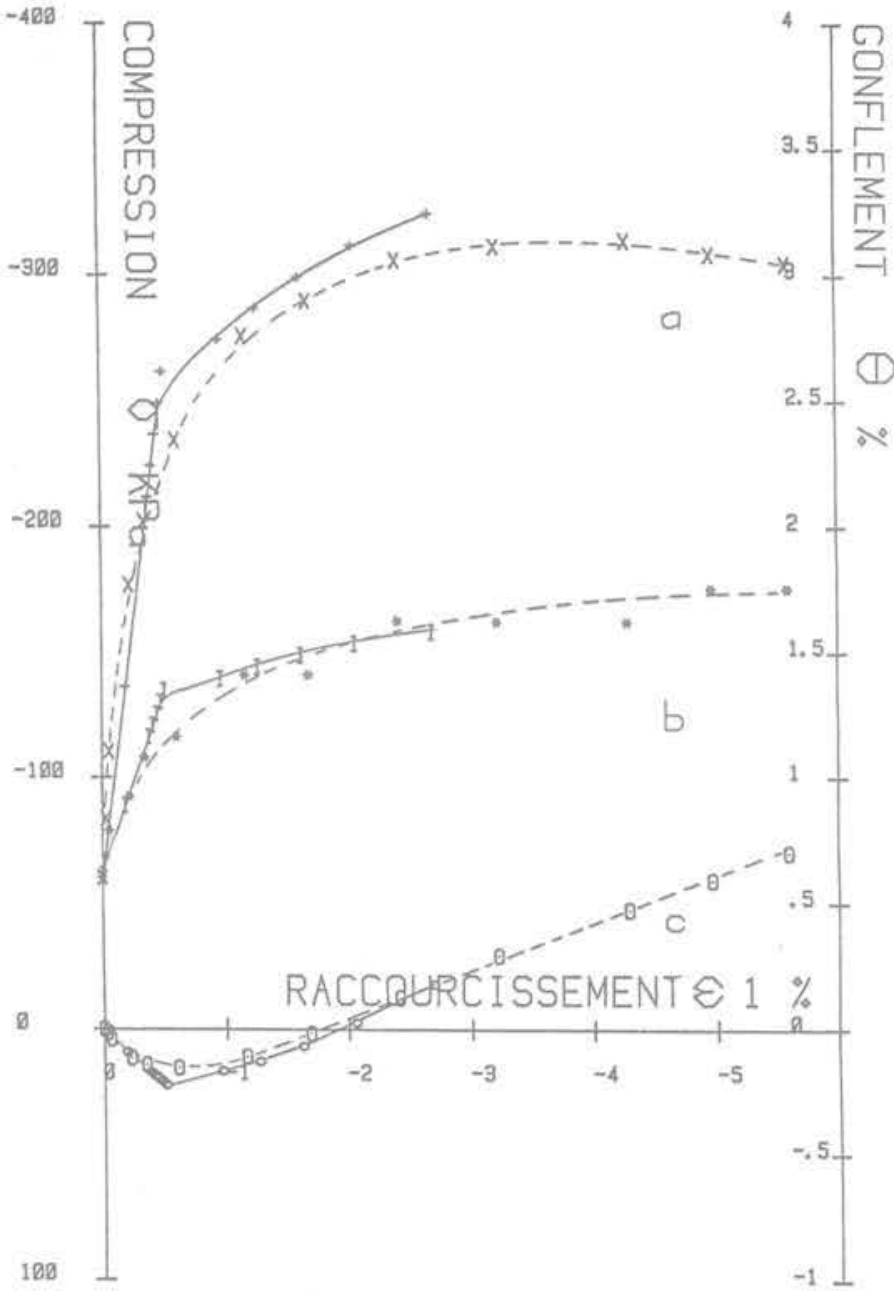

Fig. 18 Comparaison du calcul et de l'expérience pour I'essai CT1 - $\angle 2$ de Lade, $\sigma_{3}=60 \mathrm{KPa}$

en déformation plane, ce qui est une amélioration par rapport à la théorie de Rowe qui utilise $\phi_{\mu}$ et $\phi_{c y}$. Elle est d'autre part plus générale que la loi élastoplastique classique car elle tient compte de l'écrouissage durcissant et de la plasticité parfaite non standard.

Toutes ces constatations permettent de conclure à la validité de la loi proposée, et présagent de son application prochaine à de nombreux problèmes de génie civil.

\section{Références bibliographiques}

1 - AL-HUSSAINI - - Influence of relative density on the strength and deformation of sand under plane strain conditions $*$.

American Society for testing and materials, 1973 , p. 332-347.

2 - AL-HUSSAINI, RADHAKRISHNAN - "Analysis of plane strain tests using the finite element methody procedings of the Symposium on the application of the F.E.M. in geotechnical engineering. Vicksburg TMissis sippi, 1972, vol. 1, p. 215-256.

3 - ARTHUR, ASSADI - "Ruptured sand sheared in plane strain ". Congrès international de Tokyo, 1977. vol. 1, p. 19-22. 
4 - BARDEN, ISMAIL, TONG - "Plane strain deformation of granular material at low and high pressures». Geotechnique, 1969, vol. 19, n4, p. 441452.

5-BOULON, CHAMBON, DARVE - "Loi rhéologique incrémentale pour les sols et application par la méthode des éléments finis». Revue Française de Géotechnique, octobre 1977, $n^{\circ} 2$, p. 7-22.

6 - CANIZO, SAGASETA - "Earth pressure of an elastoplastic soil upon a moving rigid wall $n .5^{\circ}$ Congrès européen de mécanique des sols, Madrid, 1972. vol. 1, p. 13-21.

7 - CORNFORTH - "Some experiments on the influence of strain conditions on the strength of sand. $"$ Géotechnique, 1964, vol. 14, n², p. 143-167.

8 - DARVE, LABANIEH, CHAMBON - - Incremental stress-strain relationship for cohesionless soil. " Proc. Numerical Method in Geomechanics. Blackburg, 1977 . n०1, p. 264-269.

9 - DUNCAN, CHANG - « Non linear analysis of stress and strain in soils. " A.S.C.E. soil mechanics, septembre 1970, SM5, p. 1629-1653.

10 - FRYDMAN, ZEITLEN, ALPAN - "The yielding behaviour of particulate media. " Can. Geot. J. 101973 , p. 341-362.

11 - FRYDMAN - "Yiedling of sand in plane strain. " A.S.C.E. GT 5, May 1974, p. 491-501.

12 - GUELLEC, HUMBERT, RICARD - "La méthode des éléments finis et le système Rosalie. - Bulletin de liaison laboratoire des Ponts et Chaussées, janv. 1976 , p. 152-162.

13-ICHIHARA, MATZUZAWA - "Application of plane strain test to earth pressure. "Congrès international de Moscou, 1973, vol. 1, p. 185-190.

14 - LEE - " Comparison of plane strain and triaxial tests on sand. " A.S.C.E. S.M. 3, May 1970, p. 901-923.
15 - LADE, DUNCAN - "Elastoplastic stress-strain theory for cohesionless soil. " A.S.C.E. G'T 10, October 1975 , p. $1037-1053$.

16 - LADE - "The stress strain characteristic of cohesionless soils. " Ph. Thesis, University of California, Berkeley, 1972.

17 - MONNET - «Détermination d'une loi d'écrouissage des sols et utilisation de la méthode des éléments finis, " Thèse doct.-ingénieur, I.N.S.A. de Lyon, 1977.

18 - MONNET, GIELLY — "Détermination d'une loi de comportement pour le cisaillement des sols pulvérulents. " Revue Française de Géotechnique, 1979, $n^{\circ} 7$, p. 45-56.

19 - ODA, KOISHIKAWA, HIGUCHI - «Experimental study of anisotropic shear strength of sand by plane strain test. "Soils and Foundations, vol. $18, n^{\circ} 1$, mars 1978, p. 24-38.

20 - ROWE - "The stress dilatancy relation for static equilibrium of an assembly of particles in contact. $x$ Proc. Roy. Soc., 1962, 269-p. 500-527.

21 - ROWE - «The relation between the shear strength of sands in triaxial compression, plane strain and direct shear. " Geotechnique, 1969, vol. 19, $n^{\circ} 1$, p. 75-86.

22- ROWE, BARDEN, LEE - " Energy components during the triaxial cell and direct shear tests.". Geotechnique, 1964, vol. $14, n^{\circ} 3$, p. 247-261.

23 - SIMPSON, WROTH - "Finite element computations for a model retaining wall in sand. " $5^{\circ}$ congrès européen de mécanique des sols, Madrid, 1972, vol. 1 , p. 85-93.

24 - SIMPSON, O'RIORDAN, CORFT - «A computer model for the analysis of ground movements in London Clays. $"$ Geotechnique 29, n², 1979, p. 149-175.

25 - VERMEER - "Double hardening model for sand." Geotechnique $28,1978, n^{\circ} 4$, p. $413-433$. 
